\title{
ARMA Models for Zero Inflated Count Time Series
}

\author{
Vurukonda Sathish $^{a}$, Siuli Mukhopadhyay ${ }^{* b}$ and Rashmi Tiwari ${ }^{b}$ \\ ${ }^{a}$ Department of Electrical Engineering \\ ${ }^{b}$ Department of Mathematics \\ Indian Institute of Technology Bombay, India
}

\begin{abstract}
Zero inflation is a common nuisance while monitoring disease progression over time. This article proposes a new observation driven model for zero inflated and over-dispersed count time series. The counts given the past history of the process and available information on covariates are assumed to be distributed as a mixture of a Poisson distribution and a distribution degenerate at zero, with a time dependent mixing probability, $\pi_{t}$. Since, count data usually suffers from overdispersion, a Gamma distribution is used to model the excess variation, resulting in a zero inflated negative binomial (NB) regression model with mean parameter $\lambda_{t}$. Linear predictors with auto regressive and moving average (ARMA) type terms, covariates, seasonality and trend are fitted to $\lambda_{t}$ and $\pi_{t}$ through canonical link generalized linear models. Estimation is done using maximum likelihood aided by iterative algorithms, such as Newton Raphson (NR) and Expectation and Maximization (EM). Theoretical results on the consistency and asymptotic normality of the estimators are given. The proposed model is illustrated using in-depth simulation studies and a dengue data set.
\end{abstract}

${ }^{*}$ Corresponding author. Department of Mathematics, Indian Institute of Technology Bombay, Mumbai 400 076, India. Email: siuli@math.iitb.ac.in 
Keywords: EM algorithm, Mixture Distribution, Model Selection, Negative Binomial, Overdispersion, Prediction

\section{Introduction}

Time series modelling of counts though a popularly researched topic, is often plagued by the problem of excess zeros. For example, during geographical mapping of the prevalence of a certain disease, it is commonly noted that some regions/areas under study report a high degree of zero disease counts. In such situations, it is difficult to understand if the observed counts, represent true disease prevalence or a spurious one (i.e., the disease may be present but was not observed/reported). However, ignoring the excess zero counts while setting up the time series model may lead to incorrect estimates and loss of significant results (Perumean-Chaney et al., 2013).

The two main methods for modeling count time series are observation driven and parameter driven models (Cox (1981)). These two modelling techniques differ from each other in the way they account for the autocorrelation in the data. While in observation driven models, the autocorrelation is modeled as a function of the past responses, a latent variable approach is used in parameter driven models. For nonnormal time series, parameter driven models were considered by West et al. (1985), Fahrmeir and Wagenpfeil (1997), Durbin and Koopman (1997, 2000), Gamerman (1998), Fahrmeir and Tutz (2001), Chiogna and Gaetan (2002), Godolphin and Triantafyllopoulos (2006), Gamerman et al. (2013), among others. To compute the posterior distributions of the parameters in such models, MCMC methods (Durbin and Koopman (1997), Shephard and Pitt (1997) and Gamerman (1998)) were frequently used for

estimation. Benjamin et al. (2003) noted that often these MCMC algorithms fail to converge resulting in poor inference and predictions. Also, in more recent times the 
particle filter algorithm (Durbin and Koopman (2012)) has been sometimes used to replace these MCMC methods. Compared to parameter driven models, the computational complexity for parameter estimation is much less in observation-driven models (Chan and Ledolter (1995), 1995; Durbin and Koopman (2000), Davis et al. (2003)). In these models, the conditional distribution for each observation given past information on responses and past and possibly present covariates is described by a generalized linear model (GLM) distribution. Partial likelihood theory combined with GLMs is used for estimation. These type of models for count time series have been used by Zeger and Qaqish (1988), Li (1994), Fokianos and Kedem (2004), Shephard (1995), Davis et al. (2003), and Beniamin et al. (2003), Fokianos et al. (2009a), Fokianos and Tjøstheim (2011), Douc et al. (2013) and Davis and Liu (2016), among others.

In contrast to a large body of work on count time series models, literature on such time series with zero inflation is still very sparse. Perumean-Chaney et al. (2013) argued the importance of using a zero inflated model, and showed that ignoring these excess zeros may lead to poor estimation and chances of statistically significant findings being ignored. Yau et al. (2004) proposed a mixed autoregressive (AR) model for zero inflated count time data and applied it to an occupational health study. The autocorrelation was modeled using a random effect. More recently, Schmidt and Pereira (2011) used parameter driven models to fit zero inflated count time data, while Yang et al. (2013) proposed a zero inflated observation driven AR model. Note, all of these zero inflated models were AR in nature, not considering the possibility of including moving average (MA) components. However, in classical time series literature we have seen that MA terms play an important role, and approximation of such terms by AR terms may lead to inaccurate predictions ( $\mathrm{Li}$ (1994)). Other than AR models, generalized autoregressive conditional heteroskedastic mod- 
els suited for zero inflated integer-valued time series have also been used by Zhu (2012) and Xu et al. (2020). Very recently, a distribution-free approach for estimation of regression parameters was studied by Ghahramani and White (2020). Other techniques, like integer autoregressive (INAR) class of models, for modelling such zero inflated count time series data modeled have also been discussed by Jazi et al. (2012) and Qi et al. (2019).

In this article, we propose a new class of observation driven models for zero inflated and overdispersed time dependent counts. Our approach combines both AR as well as MA components in the zero inflated count time series model. The counts conditioned on the past information on responses and past and possibly present covariates were assumed to follow a mixture of a Poisson distribution and a distribution degenerating at zero. Since, the assumption of equality of mean and variance as made by the Poisson distribution rarely holds true in practical situations, the excess variation is modeled by a Gamma distribution, resulting in a zero inflated negative binomial (NB) model. Regression models with ARMA terms, along with trend, seasonality and various other covariates are fitted to the mean parameter of the NB, $\lambda_{t}$, and the mixing parameter, $\pi_{t}$, using log and logit links, respectively. The Newton-Raphson (NR) algorithm is used to maximize the conditional log-likelihood function and obtain the maximum likelihood (ML) estimators of the parameters. Due to the complicated expression of the log likelihood, we also develop an ExpectationMaximization (EM) algorithm to provide alternative parameter estimates. The proposed model estimators are shown to be consistent and asymptotically normal using the central limit theorem for martingales. Note for count time series ARMA models, no such asymptotic results exist in the available literature.

To motivate the necessity of including both AR and MA terms, we use our proposed approach to model the dynamics of dengue in one of the most densely pop- 
ulated cities in India. We show that the zero inflated NB-ARMA model helps us to visualise and understand the progression of dengue over time more accurately than other models with only AR terms. Since, dengue incidence has been related to various climatic factors (Jain et al. (2019), Siriyasatien et al. (2016)), we include the climatic factors, relative humidity, amount of rainfall, temperature in our model. A superior predictive performance of our proposed model when compared with various other available techniques for modelling dengue, establishes the need for using a zero inflated NB-ARMA regression model. We also fit our proposed model to the popular syphilis data (see Ghahramani and White (2020)), and compare our model's performance with other methods available in the statistical literature.

To summarise, the original contributions of this article include (i) a new ARMA type model for a zero inflated and overdispersed count time series setup, (ii) theoretical proofs of asymptotic properties of the model estimators and (iii) in-depth analysis of an unpublished Indian dengue data.

The rest of the paper is organized as follows. The proposed model is discussed in Section 2. In Section 3 we give the details on model estimation, inference and asymptotic theory. Simulation results for evaluating the estimation techniques are provided in Section 4, followed by a detailed analysis of the zero inflated dengue data in Section 5 and syphilis data in Section 6. 


\section{Proposed Statistical Model}

We model the conditional distribution of the counts at time $t, Y_{t}$, given $H_{t}$ and $w$, by a hierarchical mixture distribution,

$$
Y_{t} \mid H_{t}, w \sim \begin{cases}0 & \text { with probability } \pi_{t} \\ \operatorname{Poisson}\left(\lambda_{t} w\right) & \text { with probability }\left(1-\pi_{t}\right)\end{cases}
$$

where $w$ follows $\operatorname{Gamma}(k, k)$. Here $H_{t}$ is the information available on responses till time $(t-1)$ and on covariates till time $t$, and $w$ represents the excess variation for $t=1, \ldots, N$. The above equation can be re-expressed as,

$$
Y_{t} \mid H_{t} \sim \begin{cases}0 & \text { with probability } \pi_{t} \\ \mathrm{NB}\left(k, \frac{1}{1+\lambda_{t} / k}\right) & \text { with probability }\left(1-\pi_{t}\right) .\end{cases}
$$

or equivalently as,

$$
Y_{t} \mid H_{t}=\left\{\begin{array}{l}
0 \quad \text { with probability } \pi_{t}+\left(1-\pi_{t}\right)\left(\frac{1}{1+\lambda_{t} / k}\right)^{k} \\
m \quad \text { with probability }\left(1-\pi_{t}\right) \frac{\Gamma(m+k)}{m ! \Gamma(k)}\left(\frac{\lambda_{t} / k}{1+\lambda_{t} / k}\right)^{m}\left(\frac{1}{1+\lambda_{t} / k}\right)^{k}
\end{array}\right.
$$

where $m=1,2, \ldots$. The conditional mean and variance of $Y_{t} \mid H_{t}$ are $E\left(Y_{t} \mid H_{t}\right)=$ $\lambda_{t}\left(1-\pi_{t}\right)=\Lambda_{t}$ and $\operatorname{Var}\left(Y_{t} \mid H_{t}\right)=\lambda_{t}\left(1-\pi_{t}\right)\left[1+\lambda_{t} \pi_{t}+\lambda_{t} / k\right]=\Psi_{t}$, respectively.

As in a standard GLM setup the means are related to the linear predictor through an invertible link function $g(\cdot)$. In the zero inflated model, there are two means, the NB mean, $\lambda_{t}$, and the Bernoulli mean (mixing parameter), $\pi_{t}$, which are respectively 
modeled as,

$$
\begin{aligned}
\lambda_{t} & =\exp \left(W_{t}\right) \\
\pi_{t} & =\frac{\exp \left(M_{t}\right)}{1+\exp \left(M_{t}\right)}
\end{aligned}
$$

The state processes (linear predictors),

$$
\begin{aligned}
& W_{t}=x_{t}^{T} \beta+Z_{t} \\
& M_{t}=u_{t}^{T} \delta+V_{t}
\end{aligned}
$$

are assumed to depend on covariates $x_{t}$ and $u_{t}$ having regression functions possibly involving:

1. Trend type terms: These regression functions have the form $x_{t}=f_{1}\left(t^{\prime} /(N-1)\right)$ and $u_{t}=f_{2}\left(t^{\prime} /(N-1)\right)$ where $f_{1}(\cdot)$ and $f_{2}(\cdot)$ are real valued functions defined on the interval $[0,1]$ and $t^{\prime}=t-1$. For example, for linear trend, $f_{1}^{T}\left(t^{\prime} /(N-1)\right)=$

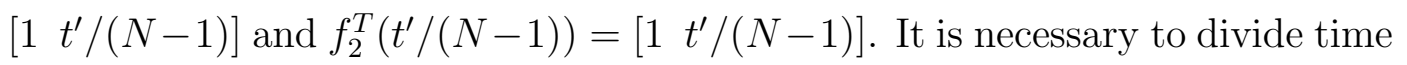
by sample size, to exclude cases where the NB (Binomial) mean may be near zero or infinity (one) for large sample sizes when trend coefficient is negative or positive, respectively.

2. Harmonic terms: These terms specify annual/half yearly/monthly/quarterly/ weekly effects. For example, $x_{t}^{T}=\left[\cos \left(2 \pi t^{\prime} / 12\right) \sin \left(2 \pi t^{\prime} / 12\right)\right]$ and $u_{t}^{T}=\left[\cos \left(2 \pi t^{\prime} / 12\right) \sin \left(2 \pi t^{\prime} / 12\right)\right]$ where $t^{\prime}=t-1$.

3. Stationary processes: These terms may specify weather series, such as humidity, rain fall, temperature etc.

Covariates $x_{t}$ and $u_{t}$ may be same or different for the two linear predictors, Autore- 
gressive and moving average terms to be added to the linear predictor are as follows: For $t \leq 0$, we define $V_{t}=Z_{t}=e_{t}=0$ and for $t>0$,

$$
\begin{aligned}
Z_{t} & =\sum_{i=1}^{p_{1}} \phi_{i}\left(Z_{t-i}+e_{t-i}\right)+\sum_{j=1}^{q_{1}} \theta_{j} e_{t-j}, \\
V_{t} & =\sum_{i=1}^{p_{2}} \alpha_{i}\left(V_{t-i}+e_{t-i}\right)+\sum_{j=1}^{q_{2}} \gamma_{j} e_{t-j},
\end{aligned}
$$

where zeros of the polynomials $\phi(z)=1-\sum_{i=1}^{p_{1}} \phi_{i} z^{i}, \theta(z)=1+\sum_{i=1}^{q_{1}} \theta_{i} z^{i}, \alpha(z)=$ $1-\sum_{i=1}^{p_{2}} \alpha_{i} z^{i}$ and $\gamma(z)=1+\sum_{i=1}^{q_{2}} \gamma_{i} z^{i}$ are outside of the unit circle. The standardized error $e_{t}$ is defined as

$$
e_{t}=\frac{\left(Y_{t}-\Lambda_{t}\right)}{\sqrt{\Psi_{t}}}
$$

The ARMA model gives $H_{t}=\left(x_{1}, \ldots, x_{t}, u_{1}, \ldots, u_{t}, y_{1}, \ldots, y_{t-1}, \Lambda_{1}, \ldots, \Lambda_{t-1}\right.$, $\left.\Psi_{1}, \ldots, \Psi_{t-1}\right)$. In general, we assume that the lengths of $x_{t}$ and $u_{t}$ are $n_{1}$ and $n_{2}$, respectively, while the unknown parameters $\beta, \phi, \theta, \delta, \alpha$ and $\gamma$ are vectors of lengths $n_{1}, p_{1}, q_{1}, n_{2}, p_{2}$ and $q_{2}$, respectively.

\subsection{Some properties of the state processes}

The ARMA models for $Z_{t}$ and $V_{t}$ can be re-written as the following $M A(\infty)$ models (Davis et al., 2003),

$$
\begin{aligned}
Z_{t} & =\sum_{j=1}^{\infty} \theta_{j} e_{t-j} \\
V_{t} & =\sum_{j=1}^{\infty} \gamma_{j} e_{t-j}
\end{aligned}
$$

Now, for $t \geq 1$, the standardized error, $e_{t}$, has expectation 0 and variance 1 . Also, the independence of $Y_{t} \mid H_{t}$ makes the errors uncorrelated, i.e., $\operatorname{Cov}\left(e_{t}, e_{s}\right)=0, t \neq s$. 
Using these error properties, we obtain,

$$
E\left(W_{t}\right)=x_{t}^{T} \beta, \quad \operatorname{Var}\left(W_{t}\right)=\sum_{j=1}^{\infty} \theta_{j}^{2}=c_{1}
$$

and, for $s=t+h, h>0, \operatorname{cov}\left(W_{t}, W_{s}\right)=\sum_{j=1}^{\infty} \theta_{j} \theta_{j+h}=c_{2}$. Similarly,

$$
E\left(M_{t}\right)=u_{t}^{T} \delta, \quad \operatorname{Var}\left(M_{t}\right)=\sum_{j=1}^{\infty} \gamma_{j}^{2}=c_{3}, \quad \operatorname{cov}\left(M_{t}, M_{s}\right)=\sum_{j=1}^{\infty} \gamma_{j} \gamma_{j+h}=c_{4}
$$

where $c_{1}, c_{2}, c_{3}$ and $c_{4}$ 's are constants. Hence, the variances and covariances of the two state processes are time independent.

For the simplest case of a MA(1) model for both $W_{t}$ and $M_{t}$, it can be shown that the bivariate Markov chain $R_{t}=\left(W_{t}, M_{t}\right)$ is bounded in probability, and therefore has at least one stationary distribution. The proof of this result is given in Supplementary materials. However, note that extension of this result to higher order models is very complex and is not shown here.

\section{Estimation}

Model fitting is done by ML using iteratively reweighted least squares, as in the context of standard GLMs. As the covariates maybe stochastic, from the conditional density of $Y_{t} \mid H_{t}$ in (3) we get the partial log-likelihood function to be (Fokianos and Kedem, 2004),

$$
\begin{aligned}
& P L(\Theta)=\sum_{t=1}^{N} P L_{t}(\Theta)=\sum_{y_{t=0}} \log \left(\pi_{t}+\left(1-\pi_{t}\right) \tilde{p}_{t}^{k}\right)+\sum_{y_{t>0}}\left[\log \left(1-\pi_{t}\right)\right. \\
& \left.+\log \Gamma\left(k+y_{t}\right)-\log \Gamma(k)-\log y_{t} !+k \log \tilde{p}_{t}+y_{t} \log \left(1-\tilde{p}_{t}\right)\right]
\end{aligned}
$$


where $\Theta=(\beta, \phi, \theta, \delta, \alpha, \gamma, k)$ is the set of all $p\left(=n_{1}+p_{1}+q_{1}+n_{2}+p_{2}+q_{2}+1\right)$ parameters. For the purpose of simplifying the notations, $\tilde{p}_{t}$ is defined to be $\frac{k}{k+\lambda_{t}}$ and $\nu=(\beta, \phi, \theta, \delta, \alpha, \gamma)$. Note, $\nu$ represents the model parameters in the processes $W_{t}$ and $M_{t}$, where

$$
\begin{aligned}
& W_{t}=x_{t}^{T} \beta+\sum_{i=1}^{p_{1}} \phi_{i}\left(Z_{t-i}+e_{t-i}\right)+\sum_{j=1}^{q_{1}} \theta_{j} e_{t-j} \\
& M_{t}=u_{t}^{T} \delta+\sum_{i=1}^{p_{2}} \alpha_{i}\left(V_{t-i}+e_{t-i}\right)+\sum_{j=1}^{q_{2}} \gamma_{j} e_{t-j},
\end{aligned}
$$

while $k$ expresses the overdispersion. The score functions are as follows:

$$
\begin{gathered}
\frac{\partial P L}{\partial \nu}=\sum_{y_{t}=0} \frac{1}{\pi_{t}+\left(1-\pi_{t}\right) \tilde{p}_{t}^{k}}\left[\frac{\partial \pi_{t}}{\partial \nu}+\left(1-\pi_{t}\right) k \tilde{p}_{t}^{k-1} \frac{\partial \tilde{p}_{t}}{\partial \nu}-\tilde{p}_{t}^{k} \frac{\partial \pi_{t}}{\partial \nu}\right] \\
+\sum_{y_{t}>0}\left[-\frac{1}{1-\pi_{t}} \frac{\partial \pi_{t}}{\partial \nu}+\frac{k}{\tilde{p}_{t}} \frac{\partial \tilde{p}_{t}}{\partial \nu}-\frac{y_{t}}{1-\tilde{p}_{t}} \frac{\partial \tilde{p}_{t}}{\partial \nu}\right], \\
\frac{\partial P L}{\partial k}=\sum_{y_{t}=0} \frac{1}{\pi_{t}+\left(1-\pi_{t}\right) \tilde{p}_{t}^{k}}\left[\frac{\partial \pi_{t}}{\partial k}+\left(1-\pi_{t}\right) \tilde{p}_{t}^{k}\left(\frac{k}{\tilde{p}_{t}} \frac{\partial \tilde{p}_{t}}{\partial k}+\log \tilde{p}_{t}\right)-\tilde{p}_{t}^{k} \frac{\partial \pi_{t}}{\partial k}\right] \\
+\sum_{y_{t}>0}\left[-\frac{1}{1-\pi_{t}} \frac{\partial \pi_{t}}{\partial k}+\psi_{0}\left(k+y_{t}\right)-\psi_{0}(k)+\frac{k}{\tilde{p}_{t}} \frac{\partial \tilde{p}_{t}}{\partial k}+\log \tilde{p}_{t}-\frac{y_{t}}{1-\tilde{p}_{t}} \frac{\partial \tilde{p}_{t}}{\partial k}\right],(12)
\end{gathered}
$$

where $\psi_{0}(\cdot)$ is a digamma function. For details see Supplementary materials.

The solutions of the score equations, $\frac{\partial P L}{\partial \nu}=0$ and $\frac{\partial P L}{\partial k}=0$ are denoted respectively by $\hat{\nu}$ and $\hat{k}$, however the estimators do not have closed form expressions necessitating the use of the NR algorithm as follows,

$$
\widehat{\Theta}^{(j+1)}=\widehat{\Theta}^{(j)}+\tilde{H}_{N}^{-1}\left(\widehat{\Theta}^{(j)}\right) S_{N}\left(\widehat{\Theta}^{(j)}\right)
$$

where $S_{N}(\Theta)=\frac{\partial P L}{\partial \Theta}$ is the score function and $\tilde{H}_{N}(\Theta)=\frac{\partial^{2} P L}{\partial \Theta \partial \Theta^{T}}$ is the observed information matrix. Detailed derivations of the components of $\tilde{H}_{n}(\Theta)$ are in Supple- 
mentary materials.

\subsection{EM algorithm}

Due to the complicated score equations, Lambert (1992) suggested an alternative use of the EM algorithm (Dempster et al. (1977)). Suppose, it is possible to observe a Bernoulli variable $S_{t}$, which takes value 1 when $Y_{t}$ is from the perfect zero state and is 0 when $Y_{t}$ is from the NB state. Then, the partial log-likelihood with complete data $\boldsymbol{y}:=\left(y_{1}, y_{2}, \ldots, y_{N}\right)$ and $\boldsymbol{s}:=\left(s_{1}, s_{2}, \ldots, s_{N}\right)$ can be written as,

$$
\begin{aligned}
& P L^{c}(\Theta)=\sum_{t=1}^{N} \log \left(f\left(s_{t} \mid H_{t}\right)\right)+\sum_{t=1}^{N} \log \left(f\left(Y_{t} \mid s_{t}, H_{t}\right)\right) \\
= & \sum_{t=1}^{N} s_{t} \log \left(\pi_{t}\right)+\left(1-s_{t}\right) \log \left(1-\pi_{t}\right) \\
+ & \sum_{t=1}^{N}\left(1-s_{t}\right)\left[\log \Gamma\left(k+y_{t}\right)-\log \Gamma(k)-\log y_{t} !+k \log \tilde{p}_{t}+y_{t} \log \left(1-\tilde{p}_{t}\right)\right]
\end{aligned}
$$

In the E-step the expectation of $P L_{t}^{c}(\Theta)$ is computed with respect to the observed count series $\boldsymbol{y}$ and current parameter estimates. The $(i+1)$ th iteration yields,

$$
\begin{aligned}
& Q\left(\Theta \mid \Theta^{(i)}\right)=E\left\{P L^{c}(\Theta) \mid \boldsymbol{y}, \Theta^{(i)}\right\}=\sum_{t=1}^{N} \hat{s}_{t}^{(i)} \log \left(\pi_{t}\right)+\left(1-\hat{s}_{t}^{(i)}\right) \log \left(1-\pi_{t}\right) \\
& \quad+\sum_{t=1}^{N}\left(1-\hat{s}_{t}^{(i)}\right)\left[\log \Gamma\left(k+y_{t}\right)-\log \Gamma(k)-\log y_{t} !+k \log \tilde{p}_{t}+y_{t} \log \left(1-\tilde{p}_{t}\right)\right]
\end{aligned}
$$




$$
\begin{aligned}
\text { where } \hat{s}_{t}^{(i)} & =\operatorname{Pr}\left(s_{t}=1 \mid Y_{t}=y_{t}, H_{t}, \Theta^{(i)}\right) \\
& =\frac{\operatorname{Pr}\left(s_{t}=1 \mid \Theta^{(i)}\right) \operatorname{Pr}\left(Y_{t}=y_{t} \mid H_{t}, s_{t}=1, \Theta^{(i)}\right)}{\operatorname{Pr}\left(Y_{t}=y_{t} \mid H_{t}, \Theta^{(i)}\right)} \\
& =\frac{\pi_{t}^{(i)} I_{\left\{y_{t}=0\right\}}}{\pi_{t}^{(i)} I_{\left\{y_{t}=0\right\}}+\left(1-\pi_{t}^{(i)}\right) \frac{\Gamma\left(y_{t}+k\right)}{y_{t} ! \Gamma(k)}\left(\frac{\lambda_{t}^{(i)} / k}{1+\lambda_{t}^{(i)} / k}\right)^{y_{t}}\left(\frac{1}{1+\lambda_{t}^{(i)} / k}\right)^{k}} .
\end{aligned}
$$

In the M-step, $\Theta^{(i+1)}$ is found by maximizing $Q\left(\Theta ; \Theta^{(i)}\right)$. The NR algorithm estimates $\Theta^{(k+1)}$ as described at the end of the previous section. The detailed derivations of the components $T_{n}(\Theta)=\frac{\partial Q\left(\Theta ; \Theta^{(i)}\right)}{\partial \Theta}$ and $J_{n}(\Theta)=\frac{\partial^{2} Q\left(\Theta ; \Theta^{(i)}\right)}{\partial \Theta \partial \Theta^{\prime}}$ are given in Supplementary materials. The sequence $\left\{\Theta_{j}: j=1,2, \ldots\right\}$ generated by the EM algorithm is a convergent sequence and it converges to local maximizer of (10).

\subsection{Asymptotic Theory}

The consistency and asymptotic normality of the maximum partial likelihood estimator $\hat{\Theta}=\left[\begin{array}{ll}\hat{\nu}^{T} & \hat{k}\end{array}\right]^{T}$ is addressed in this section. We rewrite the score functions (11) as $S_{N}(\nu)=\sum_{t=1}^{N} S^{t}(\nu)$ and $S_{N}(k)=\sum_{t=1}^{N} S^{t}(k)$ where

$$
\begin{aligned}
S^{t}(\nu) & =\frac{y_{0 t}}{\pi_{t}+\left(1-\pi_{t}\right) \tilde{p}_{t}^{k}}\left[\frac{\partial \pi_{t}}{\partial \nu}+\left(1-\pi_{t}\right) k \tilde{p}_{t}^{k-1} \frac{\partial \tilde{p}_{t}}{\partial \nu}-\tilde{p}_{t}^{k} \frac{\partial \pi_{t}}{\partial \nu}\right] \\
& +\left(1-y_{0 t}\right)\left[-\frac{1}{1-\pi_{t}} \frac{\partial \pi_{t}}{\partial \nu}+\frac{k}{\tilde{p}_{t}} \frac{\partial \tilde{p}_{t}}{\partial \nu}-\frac{y_{t}}{1-\tilde{p}_{t}} \frac{\partial \tilde{p}_{t}}{\partial \nu}\right]
\end{aligned}
$$

and

$$
\begin{gathered}
S^{t}(k)=\frac{y_{0 t}}{\pi_{t}+\left(1-\pi_{t}\right) \tilde{p}_{t}^{k}}\left[\frac{\partial \pi_{t}}{\partial k}+\left(1-\pi_{t}\right) \tilde{p}_{t}^{k}\left(\frac{k}{\tilde{p}_{t}} \frac{\partial \tilde{p}_{t}}{\partial k}+\log \tilde{p}_{t}\right)-\tilde{p}_{t}^{k} \frac{\partial \pi_{t}}{\partial k}\right] \\
+\left(1-y_{0 t}\right)\left[-\frac{1}{1-\pi_{t}} \frac{\partial \pi_{t}}{\partial k}+\psi_{0}\left(k+y_{t}\right)-\psi_{0}(k)+\frac{k}{\tilde{p}_{t}} \frac{\partial \tilde{p}_{t}}{\partial k}+\log \tilde{p}_{t}-\frac{y_{t}}{1-\tilde{p}_{t}} \frac{\partial \tilde{p}_{t}}{\partial k}\right]
\end{gathered}
$$


here $y_{0 t}:=I\left(y_{t}=0\right)$ is an indicator function. Let us consider $E\left[S^{t}(\nu) \mid H_{t}\right]$ and $E\left[S^{t}(k) \mid H_{t}\right]$ which help in proving the consistency and asymptotic normality of the maximum partial likelihood estimator $\hat{\Theta}$.

$$
\begin{aligned}
E\left[S^{t}(\nu) \mid H_{t}\right]= & E\left\{\frac{y_{0 t}}{\pi_{t}+\left(1-\pi_{t}\right) \tilde{p}_{t}^{k}}\left[\frac{\partial \pi_{t}}{\partial \nu}+\left(1-\pi_{t}\right) k \tilde{p}_{t}^{k-1} \frac{\partial \tilde{p}_{t}}{\partial \nu}-\tilde{p}_{t}^{k} \frac{\partial \pi_{t}}{\partial \nu}\right]\right. \\
& \left.+\left(1-y_{0 t}\right)\left[-\frac{1}{1-\pi_{t}} \frac{\partial \pi_{t}}{\partial \nu}+\frac{k}{\tilde{p}_{t}} \frac{\partial \tilde{p}_{t}}{\partial \nu}-\frac{y_{t}}{1-\tilde{p}_{t}} \frac{\partial \tilde{p}_{t}}{\partial \nu}\right] \mid H_{t}\right\} \\
= & \frac{E\left[y_{0 t} \mid H_{t}\right]}{\pi_{t}+\left(1-\pi_{t}\right) \tilde{p}_{t}^{k}}\left[\frac{\partial \pi_{t}}{\partial \nu}+\left(1-\pi_{t}\right) k \tilde{p}_{t}^{k-1} \frac{\partial \tilde{p}_{t}}{\partial \nu}-\tilde{p}_{t}^{k} \frac{\partial \pi_{t}}{\partial \nu}\right] \\
& +E\left[\left(1-y_{0 t}\right) \mid H_{t}\right]\left[-\frac{1}{1-\pi_{t}} \frac{\partial \pi_{t}}{\partial \nu}+\frac{k}{\tilde{p}_{t}} \frac{\partial \tilde{p}_{t}}{\partial \nu}\right]-\frac{E\left[y_{t} \mid H_{t}\right]}{1-\tilde{p}_{t}} \frac{\partial \tilde{p}_{t}}{\partial \nu} .
\end{aligned}
$$

Substituting $E\left[y_{0 t} \mid H_{t}\right]=\pi_{t}+\left(1-\pi_{t}\right) \tilde{p}_{t}^{k}, E\left[\left(1-y_{0 t}\right) \mid H_{t}\right]=\left(1-\pi_{t}\right)\left(1-\tilde{p}_{t}^{k}\right)$ and $E\left[y_{t} \mid H_{t}\right]=\lambda_{t}\left(1-\pi_{t}\right)$ in (17) above, we obtain

$$
\begin{aligned}
E\left[S^{t}(\nu) \mid H_{t}\right]= & \frac{\partial \pi_{t}}{\partial \nu}+\left(1-\pi_{t}\right) k \tilde{p}_{t}^{k-1} \frac{\partial \tilde{p}_{t}}{\partial \nu}-\tilde{p}_{t}^{k} \frac{\partial \pi_{t}}{\partial \nu} \\
& +\left(1-\pi_{t}\right)\left(1-\tilde{p}_{t}^{k}\right)\left[-\frac{1}{1-\pi_{t}} \frac{\partial \pi_{t}}{\partial \nu}+\frac{k}{\tilde{p}_{t}} \frac{\partial \tilde{p}_{t}}{\partial \nu}\right]-\frac{\lambda_{t}\left(1-\pi_{t}\right)}{1-\tilde{p}_{t}} \frac{\partial \tilde{p}_{t}}{\partial \nu} \\
= & 0 .
\end{aligned}
$$

Also,

$$
\begin{array}{r}
E\left[S^{t}(k) \mid H_{t}\right]=E\left\{\frac{y_{0 t}}{\pi_{t}+\left(1-\pi_{t}\right) \tilde{p}_{t}^{k}}\left[\frac{\partial \pi_{t}}{\partial k}+\left(1-\pi_{t}\right) \tilde{p}_{t}^{k}\left(\frac{k}{\tilde{p}_{t}} \frac{\partial \tilde{p}_{t}}{\partial k}+\log \tilde{p}_{t}\right)-\tilde{p}_{t}^{k} \frac{\partial \pi_{t}}{\partial k}\right]\right. \\
\left.+\left(1-y_{0 t}\right)\left[-\frac{1}{1-\pi_{t}} \frac{\partial \pi_{t}}{\partial k}+\psi_{0}\left(k+y_{t}\right)-\psi_{0}(k)+\frac{k}{\tilde{p}_{t}} \frac{\partial \tilde{p}_{t}}{\partial k}+\log \tilde{p}_{t}-\frac{y_{t}}{1-\tilde{p}_{t}} \frac{\partial \tilde{p}_{t}}{\partial k}\right] \mid H_{t}\right\}, \\
=\frac{E\left[y_{0 t} \mid H_{t}\right]}{\pi_{t}+\left(1-\pi_{t}\right) \tilde{p}_{t}^{k}}\left[\frac{\partial \pi_{t}}{\partial k}+\left(1-\pi_{t}\right) \tilde{p}_{t}^{k}\left(\frac{k}{\tilde{p}_{t}} \frac{\partial \tilde{p}_{t}}{\partial k}+\log \tilde{p}_{t}\right)-\tilde{p}_{t}^{k} \frac{\partial \pi_{t}}{\partial k}\right] \\
+E\left[\left(1-y_{0 t}\right) \mid H_{t}\right]\left[-\frac{1}{1-\pi_{t}} \frac{\partial \pi_{t}}{\partial k}+\frac{k}{\tilde{p}_{t}} \frac{\partial \tilde{p}_{t}}{\partial k}+\log \tilde{p}_{t}\right] \\
+E\left[\left(1-y_{0 t}\right)\left[\psi_{0}\left(k+y_{t}\right)-\psi_{0}(k)\right] \mid H_{t}\right]-\frac{E\left[y_{t} \mid H_{t}\right]}{1-\tilde{p}_{t}} \frac{\partial \tilde{p}_{t}}{\partial k} .
\end{array}
$$


Substituting $E\left[y_{0 t} \mid H_{t}\right], E\left[\left(1-y_{0 t}\right) \mid H_{t}\right]$ and $E\left[y_{t} \mid H_{t}\right]$ we get,

$$
\begin{aligned}
E\left[S^{t}(k) \mid H_{t}\right] & =\left(1-\pi_{t}\right) \log \tilde{p}_{t}+E\left[\left(1-y_{0 t}\right)\left[\psi_{0}\left(k+y_{t}\right)-\psi_{0}(k)\right] \mid H_{t}\right] \\
& =\left(1-\pi_{t}\right) \log \tilde{p}_{t}+E\left[\left(1-y_{0 t}\right) \sum_{l=1}^{y_{t}-1}\left(\frac{1}{k+l-1}\right) \mid H_{t}\right] \\
& =\left(1-\pi_{t}\right) \log \tilde{p}_{t}+E\left[\left(1-y_{0 t}\right) \log \left(1+\frac{2 y_{t}}{2 k-1}\right) \mid H_{t}\right]
\end{aligned}
$$

The second part $E\left[\left(1-y_{0 t}\right) \log \left(1+\frac{2 y_{t}}{2 k-1}\right) \mid H_{t}\right]$ in (20) is complicated. Thus, the consistency and asymptotic normality of the maximum partial likelihood estimator $\hat{\nu}$ with known overdispersion parameter $k$ is addressed in this section.

Let $S_{1}^{t}:=\frac{1}{\pi_{t}+\left(1-\pi_{t}\right) \tilde{p}_{t}^{k}}\left[\frac{\partial \pi_{t}}{\partial \nu}+\left(1-\pi_{t}\right) k \tilde{p}_{t}^{k-1} \frac{\partial \tilde{p}_{t}}{\partial \nu}-\tilde{p}_{t}^{k} \frac{\partial \pi_{t}}{\partial \nu}\right]$ and $S_{2}^{t}:=-\frac{1}{1-\pi_{t}} \frac{\partial \pi_{t}}{\partial \nu}+\frac{k}{\tilde{p}_{t}} \frac{\partial \tilde{p}_{t}}{\partial \nu}$. Then, the conditional information matrix is

$$
\begin{aligned}
G_{N}(\nu) & =\sum_{t=1}^{N} \operatorname{Var}\left[S^{t}(\nu) \mid H_{t}\right] \\
& =\sum_{t=1}^{N} \operatorname{Var}\left[y_{0 t} S_{1}^{t}+\left(1-y_{0 t}\right) S_{2}^{t}-\frac{y_{t}}{1-\tilde{p}_{t}} \frac{\partial \tilde{p}_{t}}{\partial \nu} \mid H_{t}\right] \\
& =\sum_{t=1}^{N}\left\{\left(S_{1}^{t}-S_{2}^{t}\right)\left(S_{1}^{t}-S_{2}^{t}\right)^{T} \operatorname{Var}\left(y_{0 t} \mid H_{t}\right)+\frac{\operatorname{Var}\left(y_{t} \mid H_{t}\right)}{1-\tilde{p}_{t}} \frac{\partial \tilde{p}_{t}}{\partial \nu}\left(\frac{\partial \tilde{p}_{t}}{\partial \nu}\right)^{T}\right\},
\end{aligned}
$$

where $\operatorname{Var}\left(y_{0 t} \mid H_{t}\right)=\left(\pi_{t}+\left(1-\pi_{t}\right) \tilde{p}_{t}^{k}\right)\left(1-\pi_{t}\right)\left(1-\tilde{p}_{t}^{k}\right)$ and $\operatorname{Var}\left(y_{t} \mid H_{t}\right)=\lambda_{t}\left(1-\pi_{t}\right)(1+$ $\left.\lambda_{t} \pi_{t}+\lambda_{t} / k\right)$. The unconditional information matrix is $F_{N}(\nu):=E\left[G_{N}(\nu)\right]$.

The consistency and the asymptotic normality of $\hat{\nu}$ for known $k$ is proved under the following assumptions, which are slight modifications of those stated in Fokianos and Kedem (1998; 2004).

A1. The true parameter $\nu$ belongs to an open set $\mathcal{N} \subseteq \mathbb{R}^{n_{1}+p_{1}+q_{1}+n_{2}+p_{2}+q_{2}}$.

A2. The linear predictors $W_{t}$ and $M_{t}$ are geometrically ergodic processes. 
A3. Using matrix notations, we write the the linear predictors $\left(W_{t}, M_{t}\right)$ as

$$
W_{t}=\tilde{W}^{\prime} \Theta_{1} \text { and } M_{t}=\tilde{M}_{t}^{\prime} \Theta_{2},
$$

where $\Theta_{1}=(\beta, \phi, \theta)$ and $\Theta_{2}=(\delta, \alpha, \gamma)$. The covariate vectors $\tilde{W}_{t}$ and $\tilde{M}_{t}$ almost surely lies in a nonrandom compact subset $\Gamma_{1} \subseteq \mathbb{R}^{n_{1}+p_{1}+q_{1}}$ and $\Gamma_{2} \subseteq$ $\mathbb{R}^{n_{2}+p_{2}+q_{2}}$, respectively. Also, $\tilde{W}_{t}^{\prime} \Theta_{1}$ and $\tilde{M}_{t}^{\prime} \Theta_{2}$ lie almost surely in the domain of their respective inverse link functions (i.e., $\log$ and logit) for all $\tilde{W}_{t} \in \Gamma_{1}$, $\tilde{M}_{t} \in \Gamma_{2}, \Theta_{1}$ and $\Theta_{2}$.

A4. The matrix $G_{N}(\nu)$ is a positive definite matrix with probability 1. Also, $G(\nu)$ (defined below equation 22) is a positive definite matrix at the true values of $\nu$ and therefore its inverse exists.

From assumption A2 and the law of large numbers for geometrically ergodic processes (see Jensen and Rahbek (2007) and Fokianos et al. (2009b)),

$$
\frac{G_{N}(\nu)}{N} \stackrel{\mathrm{P}}{\longrightarrow} G(\nu)
$$

as $N \rightarrow \infty$. Thus, the conditional information matrix $G_{N}(\nu)$ has a non-random limit $G(\nu)$. Here $G(\nu):=\lim _{N \rightarrow \infty} \frac{1}{N} \sum_{t=1}^{N}\left\{\left(S_{1}^{t}-S_{2}^{t}\right)\left(S_{1}^{t}-S_{2}^{t}\right)^{T} \operatorname{Var}\left(y_{0 t} \mid H_{t}\right)\right.$ $\left.+\frac{\operatorname{Var}\left(y_{t} \mid H_{t}\right)}{1-\tilde{p}_{t}} \frac{\partial \tilde{p}_{t}}{\partial \nu}\left(\frac{\partial \tilde{p}_{t}}{\partial \nu}\right)^{T}\right\}=E\left[\left(S_{1}^{t}-S_{2}^{t}\right)\left(S_{1}^{t}-S_{2}^{t}\right)^{T} \operatorname{Var}\left(y_{0 t} \mid H_{t}\right)+\frac{\operatorname{Var}\left(y_{t} \mid H_{t}\right)}{1-\tilde{p}_{t}} \frac{\partial \tilde{p}_{t}}{\partial \nu}\left(\frac{\partial \tilde{p}_{t}}{\partial \nu}\right)^{T}\right]$, for geometrically ergodic processes (see Jensen and Rahbek (2007) and Fokianos et al. $(2009 b))$.

Theorem 1. Consider the state processes, $W_{t}$ and $M_{t}$ in (4) and suppose assumptions A1-A4 hold true. Then, $S_{N}(\nu)$ converges in distribution to a normal distribution, $\mathcal{N}(0, G(\nu))$, as $N \rightarrow \infty$. 
Proof. The central limit theorem (CLT) for martingales (Corollary 3.1 of Hall and Heyde (2014)) will be used to prove the asymptotic normality of $S_{N}(\nu)$. To use the corollary, we first need to verify the following:

i. The score function is a martingale difference sequence i.e., $E\left[S^{t}(\nu) \mid H_{t}\right]=0$ and $E\left[\left\|S^{t}(\nu)\right\|\right]<\infty$ where $\|$.$\| is a vector norm (see page 230$ of Davidson (1994)),

ii. Lindeberg's condition i.e., for all $\epsilon>0$, $\frac{1}{N} \sum_{t=1}^{N} E\left[\left\|S^{t}(\nu)\right\|^{2} I\left(\left\|S^{t}(\nu)\right\|>\epsilon\right) \mid H_{t}\right] \stackrel{P}{\longrightarrow} 0$, as $N \rightarrow \infty$, and

iii. The normalized conditional information matrix $\frac{G_{N}(\nu)}{N}$ converges in probability to a non-random limit $G(\nu)$.

From (18), we know that $E\left[S^{t}(\nu) \mid H_{t}\right]=0$. Next consider,

$$
\begin{aligned}
E\left[\left\|S^{t}(\nu)\right\|\right] \leq & E\left\|\frac{y_{0 t}}{\pi_{t}+\left(1-\pi_{t}\right) \tilde{p}_{t}^{k}}\left[\frac{\partial \pi_{t}}{\partial \nu}+\left(1-\pi_{t}\right) k \tilde{p}_{t}^{k-1} \frac{\partial \tilde{p}_{t}}{\partial \nu}-\tilde{p}_{t}^{k} \frac{\partial \pi_{t}}{\partial \nu}\right]\right\| \\
& +E\left\|\left(1-y_{0 t}\right)\left[-\frac{1}{1-\pi_{t}} \frac{\partial \pi_{t}}{\partial \nu}+\frac{k}{\tilde{p}_{t}} \frac{\partial \tilde{p}_{t}}{\partial \nu}-\frac{y_{t}}{1-\tilde{p}_{t}} \frac{\partial \tilde{p}_{t}}{\partial \nu}\right]\right\| \\
\leq & E\left[\frac{y_{0 t}}{\pi_{t}+\left(1-\pi_{t}\right) \tilde{p}_{t}^{k}}\right] E\left\|\frac{\partial \pi_{t}}{\partial \nu}+\left(1-\pi_{t}\right) k \tilde{p}_{t}^{k-1} \frac{\partial \tilde{p}_{t}}{\partial \nu}-\tilde{p}_{t}^{k} \frac{\partial \pi_{t}}{\partial \nu}\right\| \\
& +E\left[1-y_{0 t}\right] E\left\|-\frac{1}{1-\pi_{t}} \frac{\partial \pi_{t}}{\partial \nu}+\frac{k}{\tilde{p}_{t}} \frac{\partial \tilde{p}_{t}}{\partial \nu}\right\|+E\left[\frac{y_{t}}{1-\tilde{p}_{t}}\right] E\left\|\frac{\partial \tilde{p}_{t}}{\partial \nu}\right\|,
\end{aligned}
$$

applying the law of total expectation to the above equation, we get

$$
\begin{aligned}
& E\left[\left\|S^{t}(\nu)\right\|\right] \leq E\left\|\frac{\partial \pi_{t}}{\partial \nu}+\left(1-\pi_{t}\right) k \tilde{p}_{t}^{k-1} \frac{\partial \tilde{p}_{t}}{\partial \nu}-\tilde{p}_{t}^{k} \frac{\partial \pi_{t}}{\partial \nu}\right\| \\
& +E\left[\left(1-\pi_{t}\right)\left(1-\tilde{p}_{t}\right)\right] E\left\|-\frac{1}{1-\pi_{t}} \frac{\partial \pi_{t}}{\partial \nu}+\frac{k}{\tilde{p}_{t}} \frac{\partial \tilde{p}_{t}}{\partial \nu}\right\|+E\left[\frac{\lambda_{t}\left(1-\pi_{t}\right)}{1-\tilde{p}_{t}}\right] E\left\|\frac{\partial \tilde{p}_{t}}{\partial \nu}\right\|
\end{aligned}
$$

Using assumptions A1 and A2 along with the fact $e_{t}=0$ for $t \leq 0$ in (4), $E\left[W_{t}\right]$ and $E\left[M_{t}\right]$ are finite, implying $E\left[\lambda_{t}\right]<\infty, E\left[\pi_{t}\right]<\infty$. Using Holders inequality 
(Hardy et al. (1934)), we can then conclude that $E\left[\left\|S^{t}(\nu)\right\|\right]<\infty$. Therefore the score function $S^{t}(\nu)$ in (15) is a martingale difference sequence.

For proving Lindeberg's condition, we consider

$$
\frac{1}{N} \sum_{t=1}^{N} E\left[\left\|S^{t}(\nu)\right\|^{2} I\left(\left\|S^{t}(\nu)\right\|>\sqrt{N} \delta\right) \mid H_{t}\right] \leq \frac{1}{N^{2} \delta^{2}} \sum_{t=1}^{N} E\left[\left\|S^{t}(\nu)\right\|^{4} \mid H_{t}\right] \stackrel{P}{\longrightarrow} 0,
$$

as $N \rightarrow \infty$, since $E\left[\left\|S^{t}(\nu)\right\|^{4}\right]<\infty$ which follows from assumptions A2, A3 and Holders inequality. The convergence in probability in (25) follows from the law of large numbers for geometrically ergodic processes (Jensen and Rahbek (2007) and Fokianos et al. (2009b)).

Next, the condition (iii) follows from (22). Therefore using the CLT for martingales, we can therefore say that the partial score function $S_{N}(\nu)$ converges in distribution to a normal random variable with zero mean and variance $G(\nu)$.

Expressing the information matrix $\tilde{H}_{N}(\nu)$ as $\tilde{H}_{N}(\nu)=G_{N}(\nu)-R_{N}(\nu)$, where $R_{N}(\nu)$ is a remainder term. Next we will show that the normalized information matrix $\frac{\tilde{H}_{N}(\nu)}{N}$ converges in probability to $G(\nu)$.

Lemma 1. Under the assumptions A1-A4, $\frac{\tilde{H}_{N}(\nu)}{N} \stackrel{P}{\rightarrow} G(\nu)$ as $N \rightarrow \infty$.

Proof. From (22), we already have $\frac{G_{N}(\nu)}{N} \stackrel{P}{\rightarrow} G(\nu)$. From assumption A2 and the law of large numbers for geometrically ergodic process (Jensen and Rahbek (2007) and Fokianos et al. (2009b)), $\frac{\tilde{H}_{N}(\nu)}{N} \stackrel{P}{\rightarrow} E\left[\frac{\partial^{2} P L_{t}(\Theta)}{\partial \nu \partial \nu^{T}}\right]$. The normalized remainder term $\frac{R_{N}(\nu)}{N}=\frac{G_{N}(\nu)}{N}+\frac{\tilde{H}_{N}(\nu)}{N}$ converges in probability to $G(\nu)+E\left[\frac{\partial^{2} P L_{t}(\Theta)}{\partial \nu \partial \nu^{T}}\right]$. Thus, we need to prove that $G(\nu)+E\left[\frac{\partial^{2} P L_{t}(\Theta)}{\partial \nu \partial \nu^{T}}\right]$ is zero. In Theorem 1 we showed that $E\left[S^{t}(\nu) \mid H_{t}\right]=0$, which implies $\sum_{t=1}^{N} S^{t}(\nu) P\left(Y_{t} \mid H_{t}\right)=0$. Differentiating both sides with respect to $\nu$ we get, $E\left[\frac{\partial^{2} P L_{t}(\Theta)}{\partial \nu \partial \nu^{T}} \mid H_{t}\right]=-E\left[S^{t}(\nu) S^{t}(\nu)^{T} \mid H_{t}\right]$. Thus, we can 
write

$$
\begin{aligned}
& G(\nu)+E\left[\frac{\partial^{2} P L_{t}(\Theta)}{\partial \nu \partial \nu^{T}}\right] \\
& \quad=E\left(\operatorname{Var}\left[S^{t}(\nu) \mid H_{t}\right]\right)+E\left[E\left[\frac{\partial^{2} P L_{t}(\Theta)}{\partial \nu \partial \nu^{T}} \mid H_{t}\right]\right] \\
& \quad=E\left(\operatorname{Var}\left[S^{t}(\nu) \mid H_{t}\right]\right)-E\left[E\left[S^{t}(\nu)\left(S^{t}(\nu)\right)^{T} \mid H_{t}\right]\right] \\
& =E\left[E\left[S^{t}(\nu)\left(S^{t}(\nu)\right)^{T} \mid H_{t}\right]-E\left[S^{t}(\nu) \mid H_{t}\right] E\left[S^{t}(\nu) \mid H_{t}\right]^{T}\right]-E\left[S^{t}(\nu)\left(S^{t}(\nu)\right)^{T}\right] \\
& =E\left[E\left[S^{t}(\nu)\left(S^{t}(\nu)\right)^{T} \mid H_{t}\right]\right]-E\left[E\left[S^{t}(\nu) \mid H_{t}\right] E\left[S^{t}(\nu) \mid H_{t}\right]^{T}\right]-E\left[S^{t}(\nu)\left(S^{t}(\nu)\right)^{T}\right] \\
& \quad=E\left[S^{t}(\nu)\left(S^{t}(\nu)\right)^{T}\right]-E\left[S^{t}(\nu)\left(S^{t}(\nu)\right)^{T}\right] \\
& =0 .
\end{aligned}
$$

Hence, the normalized remainder term $\frac{R_{N}(\nu)}{N}$ converges in probability to zero, and the normalized information matrix $\frac{\tilde{H}_{N}(\nu)}{N}$ converges in probability to $G(\nu)$, as $N \rightarrow$ $\infty$.

Lemma 2. Under the assumptions A1-A4, $\lambda_{\min }\left(F_{N}(\nu)\right) \rightarrow \infty$, as $N \rightarrow \infty$, where $\lambda_{\min }\left(F_{N}(\nu)\right)$ is the minimum eigenvalue of the unconditional information matrix $F_{N}(\nu)$

Proof is similar to that of Lemma 3.2 of Fokianos and Kedem (1998).

Theorem 2. Under the assumptions A1-A4, the probability that a locally unique maximum partial likelihood estimator exists converges to one. Also, the maximum partial likelihood estimator $\hat{\nu}$ is consistent and

$$
\sqrt{N}(\hat{\nu}-\nu) \stackrel{D}{\longrightarrow} \mathcal{N}_{n_{1}+p_{1}+q_{1}+n_{2}+p_{2}+q_{2}}\left(0, G^{-1}(\nu)\right)
$$

as $N \rightarrow \infty$. 
The outline of the proof is similar to the one in Fokianos and Kedem 1998; 2004. The details are given in Appendix A.

\subsection{Model Inference and Goodness-of-Fit Statistics}

For inferring about the model parameters, we make the assumption that the central limit theorem holds for $\widehat{\Theta}$ (for CLT for $\hat{\nu}$ see Theorem 3 ) so that, $\sqrt{n}(\widehat{\Theta}-$ $\Theta) \stackrel{\mathrm{d}}{\rightarrow} N(0, \hat{\Omega})$, as $n \rightarrow \infty$, where $\hat{\Omega}$ is the approximate covariance matrix, i.e., $\hat{\Omega}=-\left(\frac{\partial^{2} P L(\hat{\Theta})}{\partial \Theta \partial \Theta^{\prime}}\right)^{-1}$ or $\hat{\Omega}=-\left(\frac{\partial^{2} P L^{c}(\hat{\Theta})}{\partial \Theta \partial \Theta^{\prime}}\right)^{-1}$.

To test the hypotheses, $H_{0}: C \Theta=\zeta$, versus $H_{1}: C \Theta \neq \zeta$, where the matrix $C$ is of order $m \times p$ and of rank $m(\leq p)$, an approximate Wald type statistic,

$$
W=(C \widehat{\Theta}-\zeta)^{T}\left[C \hat{\Omega} C^{T}\right]^{-1}(C \widehat{\Theta}-\zeta) \sim \chi_{m}^{2} \text {, for large } N \text { under } H_{0},
$$

is used. Consider two zero inflated NB-ARMA models, such that the number of fitted parameters are $\left(m_{p_{1}}, m_{p_{2}}\right)$ and their respective deviances are, $\left(D_{m_{p_{1}}}, D_{m_{p_{2}}}\right)$. The likelihood ratio test statistic $L=D_{m_{p_{1}}}-D_{m_{p_{2}}}$ for testing between the two models may be assumed to follow a $\chi_{m_{p_{1}}-m_{p_{2}}}^{2}$ under $H_{0}$. Other than the deviance, the mean squared error (MSE), the Pearson chi-square, the two information criteria (AIC, BIC), and mean absolute deviation (MAD) may also be used to evaluate and select competing models. For definitions of these various selection criteria, refer to Chapter 1 of Kedem and Fokianos (2002).

\section{Simulation Results}

In this section the estimators and their asymptotic properties are evaluated through simulations for various zero inflated NB ARMA models. 
The models considered are:

- Model 1: Only MA terms are considered in the log and logit mean models:

$$
W_{t}=x_{t}^{T} \beta+\theta_{1} e_{t-1}+\theta_{2} e_{t-2} \quad \text { and } \quad M_{t}=u_{t}^{T} \delta+\gamma_{1} e_{t-1}
$$

- Model 2: Both AR and MA components are considered in the log and logit mean models:

$$
\begin{aligned}
& W_{t}=x_{t}^{T} \beta+\phi_{1}\left(Z_{t-1}+e_{t-1}\right)+\theta_{1} e_{t-1}+\theta_{2} e_{t-2} \text { and } \\
& M_{t}=u_{t}^{T} \delta+\alpha_{1}\left(Z_{t-1}+e_{t-1}\right)+\gamma_{1} e_{t-1}
\end{aligned}
$$

where $x_{t}^{T}=\left[\begin{array}{lll}1 & \cos (2 \pi t / 6) & \sin (2 \pi t / 6)\end{array}\right]$ and $u_{t}^{T}=\left[\begin{array}{lll}1 & \cos (2 \pi t / 6) & \sin (2 \pi t / 6)\end{array}\right]$ in both models 1 and 2 .

- Model 3: Only MA terms are considered in the log mean model:

$$
W_{t}=x_{t}^{T} \beta+\theta_{1} e_{t-1}+\theta_{3} e_{t-3} \quad \text { and } \quad M_{t}=u_{t}^{T} \delta
$$

where $x_{t}^{T}=\left[1 t^{\prime} /(N-1) \cos \left(2 \pi t^{\prime} / 52\right) \sin \left(2 \pi t^{\prime} / 52\right)\right]$ and $u_{t}^{T}=\left[1 \cos \left(2 \pi t^{\prime} / 52\right) \sin \left(2 \pi t^{\prime} / 52\right)\right]$.

Simulations with 1000 replications and sample sizes $N=30,100,500$ were run, and the results corresponding to the model 3 are displayed in Table 1, Tables for models 1 and 2 are put in the Supplementary materials. Goodness of fit plots for all models and $n=100$ are presented in Figure 1, From this figure, we note that fitted counts from both EM and MLE are very close to the observed counts.

For EM estimates (all models), we see that the average bias of the model parameters range between $(2 \%-24 \%)$ when $N=30$. As we increase sample size to 
$N=500$, the average bias reduces to $(0.07 \%-3 \%)$. The standard errors range from $(0.2 \%-1 \%)$ for all values of $N$. The results for MLE are similar. We also note from the simulation results that the bias of the overdispersion parameter $k$ is always positive and are also quite high as compared to the model parameters.

The overestimation of $k$ has been observed before in the statistical literature, see

Piegorsch (1990) and has been attributed to small values of mean parameters and sample sizes. For improving the bias, it is suggested to estimate the reciprocal of $k$ instead of $k$ itself, this is done to avoid the estimation of a discontinuous variable and also the confidence intervals of $1 / k$ are more continuous and symmetric. Also, method of moments estimation and maximum quasi likelihood estimation methods have shown to give better estimates for $k$ (Clark and Perry (1989)).

In order to check the asymptotic normality of the MLE estimators, normal probability plots (QQ plots) for Model 3 parameters are provided (see Figure 2). We see that the estimated and asymptotic densities of the model parameters $\nu$ are in agreement with each other.

\section{Real Data Analysis}

Dengue is a life-threatening mosquito-borne viral infection transmitted to humans through the bites of infected Aedes aegypti mosquitoes. The dengue virus comprises of four distinct but closely related serotypes (DEN 1-4). It is one of the most severe health problems being faced globally, with WHO reporting that there are 390 million dengue infections per year worldwide. In 2015, India alone reported 99913 dengue cases including 200 deaths. The dengue data under investigation consist of weekly dengue counts in a three year period, January 2013 to November 2015, collected from the various divisions of an Indian metro city by the public health department. 
However, the distribution of disease counts across divisions are quite uneven with some of them reporting excessive zero weekly counts (a maximum of 50\%) in the span of three years as compared to the rest. We suspect that these zero disease counts are due to underreporting of dengue cases from the divisions. Shepard et al. (2014) reports a prevalence of severe underreporting of dengue cases in India. Note the dengue data set is new and unpublished.

\subsection{An Initial Look at the Dengue Data}

Taking a preliminary glance at the dengue data we note that the data set consists of weekly counts spanning over three years. The main part of the city is divided into several administrative divisions, which are numbered alphabetically from A-F. Few of these divisions (A-D) report a high quantity of zero dengue counts, while the rest report mostly non-zero counts consistently throughout the year. We suspect that the zero dengue counts in divisions A-D arise due to their large distance from the national sentinel dengue surveillance hospitals, which leads to an under/nonreporting of active dengue cases. In this article, we analyze the dengue counts from division A, the histogram in Figure 3 shows division A has a high percentage of zeros (66 in total, i.e., $44.29 \%)$.

The distribution of weekly dengue counts (test results based on the MAC ELISA method) from division A in the period January 2013 - November 2015 (149 weeks) (see Figure 3) shows an increasing trend and yearly seasonality with high dengue counts reported during monsoon months. This non-stationary behaviour of the dengue time series is also apparent from the ACF and PACF (see Figure 44). 


\subsection{Analyzing the Dengue Data}

The proposed zero inflated NB-ARMA model for various values of the pairs $\left(p_{1}, q_{1}\right)$ and $\left(p_{2}, q_{2}\right)$ is fitted to the dengue data. The NB mean $\lambda_{t}$ is modeled using a log model, while a logit model is fitted to $\pi_{t}$. Due to the nonstationary behaviour detected in the dengue count data (see Figure 4), we tested for presence of trend and seasonality. A linear trend and yearly seasonality (represented by a pair of sine and cosine terms) were found to be statistically significant. The weather covariates introduced in the mean models were, average temperature $\left(T_{\text {avg }}\right)$, relative humidity (HMD) and rainfall (RF). However, from preliminary analysis (not reported here) it was seen that $\pi_{t}$ was not affected significantly by any of the weather covariates or any order of AR or MA terms. Based on these observations, we considered the following model forms for $W_{t}$ and $M_{t}$ (note that in each model $M_{t}$ has a simple form with only trend and seasonality terms):

\section{Model 1:}

$$
W_{t}=x_{t}^{T} \beta+\sum_{j=1}^{5} \theta_{j} e_{t-j}, \quad M_{t}=u_{t}^{T} \delta,
$$

where, $x_{t}^{T}=\left[1 t^{\prime} /(N-1) \cos \left(2 \pi t^{\prime} / 52\right), \sin \left(2 \pi t^{\prime} / 52\right) R F H M D T_{\text {avg }}\right]$ and $u_{t}^{T}=\left[1 t^{\prime} /(N-1) \cos \left(2 \pi t^{\prime} / 52\right) \sin \left(2 \pi t^{\prime} / 52\right)\right]$, here $t^{\prime}:=(t-1)$ to make the range of trend regressor $\left(t^{\prime} /(N-1)\right)$ lie in the unit interval $[0,1]$.

\section{Model 2:}

$$
W_{t}=x_{t}^{T} \beta+\sum_{j=1}^{4} \theta_{j} e_{t-j}, \quad M_{t}=u_{t}^{T} \delta
$$

\section{Model 3:}

$$
W_{t}=x_{t}^{T} \beta+\sum_{i=1}^{5} \phi_{i} Z_{t-i}+\sum_{j=1}^{5} \theta_{j} e_{t-i}, \quad M_{t}=u_{t}^{T} \delta,
$$




\section{Model 4:}

$$
W_{t}=x_{t}^{T} \beta+\sum_{j=1}^{3} \theta_{j} e_{t-j}, \quad M_{t}=u_{t}^{T} \delta
$$

for models $2-4, x_{t}^{T}=\left[1 t^{\prime} /(N-1) \cos \left(2 \pi t^{\prime} / 52\right) \sin \left(2 \pi t^{\prime} / 52\right) H M D T_{\text {avg }}\right]$ and $u_{t}^{T}=$ $\left[1 t^{\prime} /(N-1) \cos \left(2 \pi t^{\prime} / 52\right) \sin \left(2 \pi t^{\prime} / 52\right)\right]$.

\section{Model 5:}

$$
W_{t}=x_{t}^{T} \beta+\theta_{1} e_{t-1}+\theta_{3} e_{t-3}, \quad M_{t}=u_{t}^{T} \delta
$$

\section{Model 6:}

$$
W_{t}=x_{t}^{T} \beta, \quad M_{t}=u_{t}^{T} \delta
$$

where $x_{t}^{T}=\left[1 t^{\prime} /(N-1) \cos \left(2 \pi t^{\prime} / 52\right) \sin \left(2 \pi t^{\prime} / 52\right) H M D\right]$,

$u_{t}^{T}=\left[1 \cos \left(2 \pi t^{\prime} / 52\right) \sin \left(2 \pi t^{\prime} / 52\right)\right]$ and for all models $(1-6)$

$e_{t}=\left(Y_{t}-\lambda_{t}\left(1-\pi_{t}\right)\right) / \sqrt{\left[\lambda_{t}\left(1-\pi_{t}\right)\left(1+\lambda_{t} \pi_{t}+\frac{\lambda_{t}}{k}\right)\right]}$.

We choose M5 as the best model based on values of AIC and BIC (see Table 2). The deviance and chi-squared values for M5 are also small when compared to models M1-M4 and M6. The parameter estimates based on EM algorithm, standard errors and p-values for the chosen model M5 are given in Table 3. No remaining pattern is detected in the ACF and PACF plots of the residuals from M5 (see Figure 5), this is also supported by a high p-value $(>0.45)$ of the Box-Ljung test (upto lag 10). The actual observed counts versus the fitted (conditional) mean $\Lambda_{t}$ plot from M5 is shown in Figure 6. The randomized quantile residual plot corresponding to M5 in Figure 5c, show no evidence of any systematic pattern among the randomized quantile residuals and also QQ plot of the quantile residuals satisfy the normality assumptions. 
Further, the average probability of an excess zero is computed as (Lambert, 1992$)$,

$$
\hat{p}_{0}=\frac{\text { Number of }\left(y_{t}=0\right) \text { observed }-\sum_{y_{t}=0}\left(\frac{\hat{k}}{\hat{k}+\hat{\lambda}_{t}}\right)^{\hat{k}}}{N}
$$

From the observed dengue counts, the fraction of zeros was found to be 0.4429 (66 zeros out of 149 counts) and from model M5 (NB part), the fraction of zeros is estimated to be is 0.2833 . Thus, the estimated average probability of an excess zero is $\hat{p}_{0}=0.1597$. It shows that the zero-inflated part of model M5 estimates these $15.97 \%$ of excess zeros along with $28.33 \%$ of zeros from the fitted NB model.

Significant $p$-values (at level of significance 10\%) obtained by running one-sided Vuong's test (Vuong (1989)) comparing M5 with various NB models (without zeroinflation), also confirms the need for fitting a zero inflated model to the dengue data.

\subsection{A Comparative Study}

Statistical modeling of dengue data is not a new topic in the biomedical literature. Some popular techniques for analyzing dengue incidence include, generalized linear models such as Poisson and NB multivariate regression models (Xu et al., 2014; Minh An and Rocklöv, 2014; Lu et al., 2009). Modelling strategies based on Gaussian assumptions to model count time data such as, ARIMA and SARIMA models with climate covariates (Luz et al., 2008; Gharbi et al., 2011; Wongkoon et al., 2012; Martinez and Silva, 2011) are also popularly used to analyse dengue prevalence. However, due to the non-negative and integer valued nature of the data, these ARIMA/SARIMA models may give undesirable results and general exponential fam-

ily formulation may be required (Cox (1981)). Temporal models, like autoregressive 
(AR) Poisson or NB distributions (Lu et al., 2009; Briët et al., 2013) have also been fitted to dengue data. However, most of these models have a simple form with at most a first order AR term on the past responses and current values of the covariates. These simple model forms are shown to be inadequate in capturing the complex disease trajectory over time in the following comparative study.

We compared the performance of M5 with some popular statistical dengue models available in the literature. The alternative models compared are zero inflated NB models with only AR terms, NB models with no zero inflation, ARIMA and SARIMA models.

- Alternative Model (AM-1): Zero inflated Negative Binomial with AR(1) terms

$$
Y_{t}\left|s_{t}, H_{t} \sim \mathrm{NB}\left(k,\left(1-s_{t}\right) \lambda_{t}\right) \quad s_{t}\right| H_{t} \sim \operatorname{Bernoulli}\left(\pi_{t}\right)
$$

The means $\lambda_{t}$ and $\omega_{t}$ are modeled using the link functions,

$$
\log \left(\lambda_{t}\right)=x_{t}^{T} \beta+\theta I_{\left\{y_{t-1}>0\right\}}, \quad \operatorname{logit}\left(\pi_{t}\right)=u_{t}^{T} \delta
$$

where $H_{t}=\left(y_{t-1}, x_{t}, u_{t}\right)$,

$$
\begin{aligned}
x_{t}^{T} & =\left[1, t^{\prime} /(N-1), \cos \left(2 \pi t^{\prime} / 52\right), \sin \left(2 \pi t^{\prime} / 52\right), H M D\right] \text { and } \\
u_{t}^{T} & =\left[1, \cos \left(2 \pi t^{\prime} / 52\right), \sin \left(2 \pi t^{\prime} / 52\right)\right] .
\end{aligned}
$$

- Alternative Model (AM-2): Negative Binomial $\left(k, \lambda_{t} /\left(\lambda_{t}+k\right)\right)$ with $\operatorname{AR}(1)$ terms

$$
\log \left(\lambda_{t}\right)=x_{t}^{T} \beta+\theta\left\{\log \left(y_{t-j}^{*}\right)-x_{t-j}^{T} \beta\right\}
$$

where $y_{t-j}^{*}=\max \left(y_{t-j}, c\right), 0<c<1$ and 


$$
x_{t}^{T}=\left[1, t^{\prime} /(N-1), \cos \left(2 \pi t^{\prime} / 52\right), \sin \left(2 \pi t^{\prime} / 52\right), H M D\right] .
$$

- Alternative Model (AM-3): ARIMA $(p, r, q)$ model was fitted to the dengue counts. The best model with least AIC and BIC values was the ARIMA $(2,0,2)$.

- Alternative Model (AM-4): The best SARIMA model with respect to AIC and BIC values was SARIMA $(2,0,0)(1,0,1)^{52}$.

Table 4 shows that M5 has the lowest values of all four reported goodness of fit statistics. By setting different threshold values (i.e., if $\hat{Y}_{t}<$ threshold value, then

assume $\hat{Y}_{t}=0$ ), we conducted a sensitivity analysis (see results in Table 5 ) of zero counts prediction by model M5 and the zero inflated model AM-1, the table shows that the accuracy of M5 identifying the zeros is larger than AM-1.

Other than the above discussed alternative models, we also used a neural network approach for dengue data fitting. Neural networks with one/two hidden layers and $(1,3,7,10)$ hidden nodes with both sigmoid and tanh activation functions were tried. The inputs were the response variables till lag 4, humidity, rainfall and average temperature. The network with one hidden layer and node and the tanh activation function gave the lowest values of MSE $=16.47$ and $\mathrm{MAD}=4.05$.

\section{Analyzing the Syphilis Data}

In this section, we fit our zero inflated NB-ARMA model to the time series of weekly syphilis disease counts in Virginia, USA between 2007 and 2010. The percentage of zeros in the syphilis data is $26.79 \%$. Our proposed model for various values of the pairs $\left(p_{1}, q_{1}\right)$ and $\left(p_{2}, q_{2}\right)$ is fitted to the syphilis data. Due to the nonstationary behaviour detected in the syphilis count data we tested and found the presence of 
a significant linear trend. Based on a comparison of MSE, AIC and BIC values we chose the following model to fit the syphilis data:

$$
W_{t}=x_{t}^{T} \beta+\sum_{j=1}^{2} \theta_{j} e_{t-j}, \quad M_{t}=u_{t}^{T} \delta
$$

where, $x_{t}^{T}=\left[\begin{array}{ll}1 & t / 1000\end{array}\right]$ and $u_{t}^{T}=\left[\begin{array}{ll}1 & t / 1000\end{array}\right]$ for all models $(1-6) e_{t}=\left(Y_{t}-\right.$ $\left.\lambda_{t}\left(1-\pi_{t}\right)\right) / \sqrt{\left[\lambda_{t}\left(1-\pi_{t}\right)\left(1+\lambda_{t} \pi_{t}+\frac{\lambda_{t}}{k}\right)\right]}$.

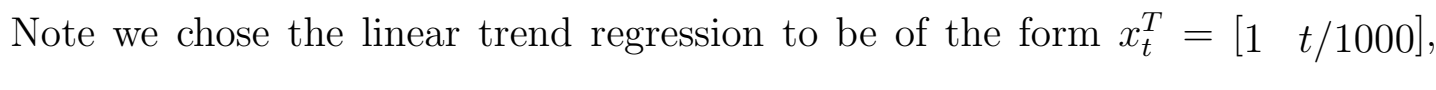
since this form has been used before in the literature (see Yang et al. (2013) and Ghahramani and White (2020)). The EM based results for the chosen model are shown in Table 7 .

We compared our model's performance with the distribution-free functional response (FRM) model of Ghahramani and White (2020), a zero inflated Poisson (ZIP), and a zero inflated NB (ZINB). The comparison is shown in Table 8, Note that the MSE, AIC and BIC values for our zero-inflated NB-ARMA model are lesser than the other models frequently used in the literature.

\section{Concluding Remarks}

In this article, we proposed a zero inflated NB-ARMA model to fit correlated count time data with excessive zeros and variation. The proposed model was successfully fitted to weekly dengue and syphilis counts with zero inflation. In a detailed comparison of the chosen dengue and syphilis models with various alternative models available in the literature we saw that the selected model had the lowest values of RMSE and MAD values. All computations were done using the $\mathrm{R}$ and Matlab statistical softwares. The programs are available on request from the first author. 
In future, we are interested in extending the proposed model to a multivariate time series setup using copulas. As an example, we may consider setting up a model for dengue counts over time simultaneously for several regions.

\section{Appendix A: Proof of Theorem 2}

We first prove asymptotic existence and consistency of maximum partial likelihood estimator $\hat{\nu}$. By Taylor expansion at true parameters $\nu$,

$$
P L(\tilde{\nu})=P L(\nu)+(\tilde{\nu}-\nu)^{T} S_{N}(\nu)-\frac{1}{2}(\tilde{\nu}-\nu)^{T} \tilde{H}_{N}(\tilde{\tilde{\nu}})(\tilde{\nu}-\nu),
$$

where $\tilde{\tilde{\nu}}$ lies between $\tilde{\nu}$ and $\nu$. Then,

$$
P L(\tilde{\nu})-P L(\nu)=(\tilde{\nu}-\nu)^{T} S_{N}(\nu)-\frac{1}{2}(\tilde{\nu}-\nu)^{T} \tilde{H}_{N}(\tilde{\nu})(\tilde{\nu}-\nu) .
$$

Let $O_{N}(\delta):=\left\{\tilde{\nu}:\left\|F_{N}(\nu)^{T / 2}(\tilde{\nu}-\nu)\right\| \leq \delta\right\}$ and $\tilde{\lambda}:=F_{N}(\nu)^{T / 2}(\tilde{\nu}-\nu) / \delta$ where $F_{N}(\nu)^{T / 2}:=\left(F_{N}(\nu)^{1 / 2}\right)^{T}$. Then, $(\tilde{\nu}-\nu)^{T}=\tilde{\lambda}^{T} F_{N}(\nu)^{-1 / 2} \delta$, (35) becomes,

$$
P L(\tilde{\nu})-P L(\nu)=\delta \tilde{\lambda}^{T} F_{N}(\nu)^{-1 / 2} S_{N}(\nu)-\frac{\delta^{2}}{2} \tilde{\lambda}^{T} F_{N}(\nu)^{-1 / 2} \tilde{H}_{N}(\tilde{\tilde{\nu}}) F_{N}(\nu)^{-T / 2} \tilde{\lambda} .
$$

We are going to prove that for every $\eta>0$ there exist $N$ and $\delta$ such that

$$
P\left[(P L(\tilde{\nu})-P L(\nu))<0 \forall \tilde{\nu} \in \partial O_{N}(\delta)\right] \geq 1-\eta,
$$

where $\partial O_{N}(\delta):=\left\{\tilde{\nu}:\left\|F_{N}(\nu)^{T / 2}(\tilde{\nu}-\nu)\right\|=\delta\right\}$ is a boundary set of $O_{N}(\delta)$. This show that with probability tending to one, there exists a local maximum inside 
$O_{N}(\delta)$. Then the left part of inequality (37) becomes,

$$
\begin{aligned}
P & {[(P L(\tilde{\nu})-P L(\nu))<0] } \\
& =P\left[\delta \tilde{\lambda}^{T} F_{N}(\nu)^{-1 / 2} S_{N}(\nu)-\frac{\delta^{2}}{2} \tilde{\lambda}^{T} F_{N}(\nu)^{-1 / 2} \tilde{H}_{N}(\tilde{\tilde{\nu}}) F_{N}(\nu)^{-T / 2} \tilde{\lambda}<0\right] \\
& =P\left[\delta \tilde{\lambda}^{T} F_{N}(\nu)^{-1 / 2} S_{N}(\nu)-\frac{\delta^{2}}{2} \tilde{\lambda}^{T} F_{N}(\nu)^{-1 / 2} \tilde{H}_{N}(\tilde{\tilde{\nu}}) F_{N}(\nu)^{-T / 2} \tilde{\lambda}<0\right]
\end{aligned}
$$

Note that $\tilde{\lambda}^{T} \tilde{\lambda}=1$. Consider

$$
\begin{aligned}
\delta \tilde{\lambda}^{T} F_{N}(\nu)^{-1 / 2} & S_{N}(\nu)-\frac{\delta^{2}}{2} \tilde{\lambda}^{T} F_{N}(\nu)^{-1 / 2} \tilde{H}_{N}(\tilde{\tilde{\nu}}) F_{N}(\nu)^{-T / 2} \tilde{\lambda} \\
& \leq \delta\left\|\tilde{\lambda} F_{N}(\nu)^{-1 / 2} S_{N}(\nu)\right\|-\frac{\delta^{2}}{2} \lambda_{\min }\left(F_{N}(\nu)^{-1 / 2} \tilde{H}_{N}(\tilde{\tilde{\nu}}) F_{N}(\nu)^{-T / 2}\right) \\
& \leq \delta\|\tilde{\lambda}\|\left\|F_{N}(\nu)^{-1 / 2} S_{N}(\nu)\right\|-\frac{\delta^{2}}{2} \lambda_{\min }\left(F_{N}(\nu)^{-1 / 2} \tilde{H}_{N}(\tilde{\tilde{\nu}}) F_{N}(\nu)^{-T / 2}\right) \\
& =\delta\left\|F_{N}(\nu)^{-1 / 2} S_{N}(\nu)\right\|-\frac{\delta^{2}}{2} \lambda_{\min }\left(F_{N}(\nu)^{-1 / 2} \tilde{H}_{N}(\tilde{\tilde{\nu}}) F_{N}(\nu)^{-T / 2}\right)
\end{aligned}
$$

Substitute (39) in (38), then (38) becomes

$$
\begin{aligned}
P & {[(P L(\tilde{\nu})-P L(\nu))<0] } \\
& =P\left[\left\|F_{N}(\nu)^{-1 / 2} S_{N}(\nu)\right\|^{2} \leq \frac{\delta^{2}}{4} \lambda_{\min }^{2}\left(F_{N}(\nu)^{-1 / 2} \tilde{H}_{N}(\tilde{\tilde{\nu}}) F_{N}(\nu)^{-T / 2}\right)\right]
\end{aligned}
$$

From Lemma 1, we know that $\frac{\tilde{H}_{N}(\nu)}{N} \stackrel{P}{\rightarrow} G(\nu)$ as $N \rightarrow \infty$. Then, the normalized information matrix $F_{N}(\nu)^{-1 / 2} \tilde{H}_{N}(\nu) F_{N}(\nu)^{-T / 2}$ converges in probability to identity matrix $I$. Note that the set $\left\{O_{N}(\delta)\right\}$ shrinks to $\nu$ as $N \rightarrow \infty$. Thus, the normalized information matrix $F_{N}(\nu)^{-1 / 2} \tilde{H}_{N}(\tilde{\tilde{\nu}}) F_{N}(\nu)^{-T / 2}$ converges in probability to identity matrix $I$ since $\tilde{\tilde{\nu}}$ goes to $\nu$ as $N \rightarrow \infty$. From Cramer-Wold Device, for any $\lambda \neq 0$, $\lambda^{T} F_{N}(\nu)^{-1 / 2} \tilde{H}_{N}(\tilde{\tilde{\nu}}) F_{N}(\nu)^{-T / 2} \lambda$ converges in probability to 1 . Then there exist $\lambda$ such that $\lambda_{\min }\left(F_{N}(\nu)^{-1 / 2} \tilde{H}_{N}(\tilde{\tilde{\nu}}) F_{N}(\nu)^{-T / 2}\right)$ converges in probability to 1 . From Lemma 
3.4 of Fokianos and Kedem (1998), the expression (40) becomes

$$
\begin{aligned}
P & {[(P L(\tilde{\nu})-P L(\nu))<0] } \\
& =P\left[\left\|F_{N}(\nu)^{-1 / 2} S_{N}(\nu)\right\|^{2} \leq \frac{\delta^{2}}{4} \lambda_{\min }^{2}\left(F_{N}(\nu)^{-1 / 2} \tilde{H}_{N}(\tilde{\tilde{\nu}}) F_{N}(\nu)^{-T / 2}\right)\right] \\
& \geq P\left[\left\|F_{N}(\nu)^{-1 / 2} S_{N}(\nu)\right\|^{2} \leq \frac{\delta^{2}}{4}\right] \\
& \geq 1-\frac{4}{\delta^{2}} E\left[\left\|F_{N}(\nu)^{-1 / 2} S_{N}(\nu)\right\|^{2}\right] .
\end{aligned}
$$

For a given $\eta>0$, choosing $\delta=\sqrt{\frac{4}{\eta} E\left[\left\|F_{N}(\nu)^{-1 / 2} S_{N}(\nu)\right\|^{2}\right]}$, then we have

$$
P\left[(P L(\tilde{\nu})-P L(\nu))<0 \forall \tilde{\nu} \in \partial O_{N}(\delta)\right] \geq 1-\eta .
$$

Therefore asymptotic existence is established. More specifically, there exists a sequence of maximum partial likelihood estimators $\hat{\nu}$, such that for any $\eta>0$, there are $\delta$ and $N_{1}$ such that

$$
\begin{aligned}
P\left[\hat{\nu} \in O_{N}(\delta)\right] & \geq 1-\eta \\
P\left[\left\|F_{N}(\nu)^{-T / 2}(\hat{\nu}-\nu)\right\| \leq \delta\right] & \geq 1-\eta \\
P\left[\|\hat{\nu}-\nu\| \leq \frac{\delta}{\lambda_{\min }\left(F_{N}(\nu)\right)}\right] & \geq 1-\eta
\end{aligned}
$$

Thus, the maximum partial likelihood estimator $\hat{\nu}$ is locally unique. Therefore consistency is established.

Next, we prove asymptotic normality. By Taylor expansion around $\hat{\nu}$, and using 
the mean value theorem for multivariate function we obtain for $0 \leq s \leq 1$,

$$
\begin{aligned}
S_{N}(\nu) & =S_{N}(\hat{\nu})+\tilde{H}_{N}(\nu+s(\hat{\nu}-\nu))(\hat{\nu}-\nu) \\
& =\tilde{H}_{N}(\nu+s(\hat{\nu}-\nu))(\hat{\nu}-\nu),
\end{aligned}
$$

where $\nu+s(\hat{\nu}-\nu)$ lies between $\hat{\nu}$ and $\nu$. Multiply $F_{N}(\nu)^{-1 / 2}$ both sides to (44), then

$$
\begin{aligned}
F_{N}(\nu)^{-1 / 2} S_{N}(\nu) & =F_{N}(\nu)^{-1 / 2} \tilde{H}_{N}(\nu+s(\hat{\nu}-\nu))(\hat{\nu}-\nu) \\
= & \left(F_{N}(\nu)^{-1 / 2} \tilde{H}_{N}(\nu+s(\hat{\nu}-\nu)) F_{N}(\nu)^{-T / 2}\right)\left(F_{N}(\nu)^{T / 2}(\hat{\nu}-\nu)\right) .
\end{aligned}
$$

From Theorem 1, The score function $S_{N}(\nu)$ converges in distribution to $\mathcal{N}(0, G(\nu))$, as $N \rightarrow \infty$. The normalized score function $F_{N}^{-1 / 2} S_{N}(\nu)$ converges in distribution to $\mathcal{N}(0, I)$. From consistency (43) and Lemma 1, $F_{N}(\nu)^{-T / 2} \tilde{H}_{N}(\nu+s(\hat{\nu}-\nu)) F_{N}(\nu)^{-T / 2}$ converges in probability to identity matrix $I$. Then, the expression (45) becomes,

$$
F_{N}(\nu)^{T / 2}(\hat{\nu}-\nu) \rightarrow \mathcal{N}(0, I)
$$

in distribution as $N \rightarrow \infty$. The normalized conditional information matrix $F_{N}(\nu)^{-1 / 2} G_{N}(\nu) F_{N}(\nu)^{-1 / 2}$ converges in probability to identity matrix $I$. Choosing $G_{N}(\nu)^{1 / 2}$ such that $F_{N}(\nu)^{-1 / 2} G_{N}(\nu)^{1 / 2}$ is the Cholesky square root of $F_{N}(\nu)^{-1 / 2} G_{N}(\nu) F_{N}(\nu)^{-1 / 2}$. We have from the continuity of the square root that $F_{N}(\nu)^{-1 / 2} G_{N}(\nu)^{1 / 2}$ converges in probability to identity matrix $I$. Thus,

$$
G_{N}(\nu)^{T / 2}(\hat{\nu}-\nu)=G_{N}(\nu)^{T / 2} F_{N}(\nu)^{-T / 2} F_{N}(\nu)^{T / 2}(\hat{\nu}-\nu) \rightarrow \mathcal{N}(0, I)
$$

as $N \rightarrow \infty$. From the continuity of the square root $\frac{G_{N}(\nu)^{T / 2}}{\sqrt{N}}$ converges in probability 
$G(\nu)^{T / 2}$. Then (47) becomes,

$$
\begin{gathered}
\sqrt{N}\left(\frac{G_{N}(\nu)^{T / 2}}{\sqrt{N}}\right)(\hat{\nu}-\nu) \rightarrow \mathcal{N}(0, I), \\
\sqrt{N}(\hat{\nu}-\nu) \rightarrow \mathcal{N}\left(0, G(\nu)^{-1}\right)
\end{gathered}
$$

in distribution as $N \rightarrow \infty$.

\section{Acknowledgements}

The work of S. Mukhopadhyay was supported by the Science and Research Engineering Board (Department of Science and Technology, Government of India) [File Number: EMR/2016/005142] and Wadhwani Research Centre for Bio-Engineering. We would like to acknowledge Professors Konstantinos Fokianos and Monika Bhattacharjee for their helpful suggestions.

\section{References}

Benjamin, M. A., R. A. Rigby, and D. M. Stasinopoulos (2003). Generalized autoregressive moving average models. Journal of the American Statistical Association 98(461), 214-223.

Briët, O. J., P. H. Amerasinghe, and P. Vounatsou (2013). Generalized seasonal autoregressive integrated moving average models for count data with application to malaria time series with low case numbers. PLoS One 8(6), e65761.

Chan, K. S. and J. Ledolter (1995). Monte Carlo EM estimation for time series 
models involving counts. Journal of the American Statistical Association 90, 242252.

Chiogna, M. and C. Gaetan (2002). Dynamic generalized linear models with application to environmental epidemiology. Applied Statistics 51, 453-468.

Clark, S. and J. Perry (1989). Estimation of the negative binomial parameter k by maximum quasilikelihood. Biometrics 45(1), 309-316.

Cox, D. R. (1981). Statistical analysis of time series: Some recent developments. Scandinavian Journal of Statistics 8, 93-115.

Davidson, J. (1994). Stochastic limit theory: An introduction for econometricians. OUP Oxford.

Davis, R. A., W. T. M. Dunsmuir, and S. B. Streett (2003). Observation-driven models for Poisson counts. Biometrika 90(4), 777-790.

Davis, R. A. and H. Liu (2016). Theory and inference for a class of observation-driven models with application to time series of counts. Statistica Sinica 102, 1673-1707.

Dempster, A. P., N. M. Laird, and D. B. Rubin (1977). Maximum likelihood from incomplete data via the EM algorithm. Journal of the Royal Statistical Society. Series B 39(1), 1-38.

Douc, R., P. Doukhan, and E. Moulines (2013). Ergodicity of observation-driven time series models and consistency of the maximum likelihood estimator. Stochastic Processes and their Applications 123, 2620- 2647.

Durbin, J. and S. J. Koopman (1997). Monte Carlo maximum likelihood estimation for non-gaussian state space models. Biometrika 84, 669-684. 
Durbin, J. and S. J. Koopman (2000). Time series analysis of non-Gaussian observations based on state space models from both classical and Bayesian perspectives. Journal of the Royal Statistical Society. Series B 62(1), 3-56. With discussion and a reply by the authors.

Durbin, J. and S. J. Koopman (2012). Time Series Analysis by State Space Methods. Oxford University Press.

Fahrmeir, L. and G. Tutz (2001). Multivariate Statistical Modeling Based on Generalized Linear Models. Springer-Verlag, New York.

Fahrmeir, L. and S. Wagenpfeil (1997). Penalized likelihood estimation and iterative Kalman filtering for non-gaussian dynamic regression models. Computational Statistics and Data Analysis 24, 295-320.

Fokianos, K. and B. Kedem (1998). Prediction and classification of non-stationary categorical time series. Journal of Multivariate Analysis 67, 277-296.

Fokianos, K. and B. Kedem (2004). Partial likelihood inference for time series following generalized linear models. Journal of Time Series Analysis 25(2), 173-197.

Fokianos, K., A. Rahbek, and D. Tjøstheim (2009a). Poisson autoregression. Journal of the American Statistical Association 104, 1430-1439.

Fokianos, K., A. Rahbek, and D. Tjøstheim (2009b). Poisson autoregression. Journal of the American Statistical Association 104(488), 1430-1439.

Fokianos, K. and D. Tjøstheim (2011). Log-linear poisson autoregression. Journal of Multivariate Analysis 102, 563-578.

Gamerman, D. (1998). Markov chain monte carlo for dynamic generalised linear models. Biometrika 85, 215-227. 
Gamerman, D., T. R. Santos, and G. C. Franco (2013). A non-gaussian family of state-space models with exact marginal likelihood. Journal of Time Series Analysis 34, 625-645.

Ghahramani, M. and S. White (2020). Time series regression for zero-inflated and overdispersed count data: A functional response model approach. Journal of Statistical Theory and Practice 14(2), 1-18.

Gharbi, M., P. Quenel, J. Gustave, S. Cassadou, G. La Ruche, L. Girdary, and L. Marrama (2011). Time series analysis of dengue incidence in Guadeloupe, French West Indies: forecasting models using climate variables as predictors. $B M C$ Infectious Diseases 11(1), 166.

Godolphin, E. J. and K. Triantafyllopoulos (2006). Decomposition of time series models in state-space form. Computational Statistics and Data Analysis 50, 22322246 .

Hall, P. and C. C. Heyde (2014). Martingale limit theory and its application. Academic press, London.

Hardy, G., J. Littlewood, and Polya (1934). Inequalities. Cambridge University Press.

Jain, R., S. Sontisirikit, S. Iamsirithaworn, and H. Prendinger (2019). Prediction of dengue outbreaks based on disease surveillance, meteorological and socio-economic data. BMC Infectious Diseases 19(1), 272.

Jazi, M. A., G. Jones, and C.-D. Lai (2012). First-order integer valued AR processes with zero inflated poisson innovations. Journal of Time Series Analysis 33(6), 954-963. 
Jensen, S. T. and A. Rahbek (2007). On the law of large numbers for (geometrically) ergodic markov chains. Econometric Theory, 761-766.

Kedem, B. and K. Fokianos (2002). Regression models for time series analysis. John Wiley \& Sons, Hoboken, New Jersey.

Lambert, D. (1992). Zero-inflated poisson regression, with an application to defects in manufacturing. Technometrics 34(1), 1-14.

Li, W. K. (1994). Time series models based on generalized linear models: Some further results. Biometrics 50(2), 506-511.

Lu, L., H. Lin, L. Tian, W. Yang, J. Sun, and Q. Liu (2009). Time series analysis of dengue fever and weather in Guangzhou, China. BMC Public Health 9(1), 395.

Luz, P. M., B. V. Mendes, C. T. Codeço, C. J. Struchiner, and A. P. Galvani (2008). Time series analysis of dengue incidence in Rio de Janeiro, Brazil. The American Journal of Tropical Medicine and Hygiene 79(6), 933-939.

Martinez, E. Z. and E. A. S. d. Silva (2011). Predicting the number of cases of dengue infection in Ribeirão Preto, São Paulo State, Brazil, using a sarima model. Cadernos de Saude Publica 27, 1809-1818.

Minh An, D. T. and J. Rocklöv (2014). Epidemiology of dengue fever in Hanoi from 2002 to 2010 and its meteorological determinants. Global Health Action 7(1), 23074 .

Perumean-Chaney, S. E., C. Morgan, D. McDowall, and I. Aban (2013). Zeroinflated and overdispersed: what's one to do? Journal of Statistical Computation and Simulation 83, 1671-1683. 
Piegorsch, W. (1990). Maximum likelihood estimation for the negative binomial dispersion parameter. Biometrics 46(3), 863-867.

Qi, X., Q. Li, and F. Zhu (2019). Modeling time series of count with excess zeros and ones based on INAR (1) model with zero-and-one inflated poisson innovations. Journal of Computational and Applied Mathematics 346, 572-590.

Schmidt, A. M. and J. B. M. Pereira (2011). Modelling time series of counts in epidemiology. International Statistical Review 79(1), 48-69.

Shepard, D. S., Y. A. Halasa, B. K. Tyagi, S. V. Adhish, D. Nandan, K. Karthiga, V. Chellaswamy, M. Gaba, N. K. Arora, I. S. Group, et al. (2014). Economic and disease burden of dengue illness in india. The American Journal of Tropical Medicine and Hygiene 91 (6), 1235-1242.

Shephard, N. (1995). Generalized linear autoregressions. Economics Papers 8., Economics Group, Nuffield College, University of Oxford.

Shephard, N. and M. K. Pitt (1997). Likelihood analysis of non-gaussian measurement time series. Biometrika 84, 653-667.

Siriyasatien, P., A. Phumee, P. Ongruk, K. Jampachaisri, and K. Kesorn (2016). Analysis of significant factors for dengue fever incidence prediction. BMC Bioinformatics 17(1), 166.

Vuong, Q. (1989). Likelihood ratio tests for model selection and non-nested hypotheses. Econometrica 57, 307-333.

West, M., P. J. Harrison, and H. S. Migon (1985). Dynamic generalized linear models and Bayesian forecasting. Journal of the American Statistical Association 80, 7396. 
Wongkoon, S., M. Jaroensutasinee, and K. Jaroensutasinee (2012). Development of temporal modeling for prediction of dengue infection in northeastern Thailand. Asian Pacific Journal of Tropical Medicine 5(3), 249-252.

Xu, H.-Y., X. Fu, L. K. H. Lee, S. Ma, K. T. Goh, J. Wong, M. S. Habibullah, G. K. K. Lee, T. K. Lim, P. A. Tambyah, et al. (2014). Statistical modeling reveals the effect of absolute humidity on dengue in Singapore. PLoS Neglected Tropical Diseases 8(5), e2805.

Xu, X., Y. Chen, C. W. Chen, X. Lin, et al. (2020). Adaptive log-linear zero-inflated generalized poisson autoregressive model with applications to crime counts. Annals of Applied Statistics 14(3), 1493-1515.

Yang, M., G. K. Zamba, and J. E. Cavanaugh (2013). Markov regression models for count time series with excess zeros: A partial likelihood approach. Statistical Methodology 14, 26-38.

Yau, K. K., A. H. Lee, and P. J. Carrivick (2004). Modeling zero-inflated count series with application to occupational health. Computer Methods and Programs in Biomedicine 74(1), 47-52.

Zeger, S. L. and B. Qaqish (1988). Markov regression models for time series: a quasi-likelihood approach. Biometrics. Journal of the Biometric Society 44(4), $1019-1031$.

Zhu, F. (2012). Zero-inflated poisson and negative binomial integer-valued GARCH models. Journal of Statistical Planning and Inference 142(4), 826-839. 
Table 1: Simulation results for Model 3 in Section 4

\begin{tabular}{|c|c|c|c|c|c|}
\hline \multicolumn{6}{|c|}{ EM } \\
\hline \multicolumn{6}{|c|}{$\mathrm{N}=30$} \\
\hline Parameters & True & Est. & S.E. & |Bias & C.I. \\
\hline Intercept & 0.3 & 0.3461 & 0.0212 & 0.0461 & $(0.3045,0.3877)$ \\
\hline$t / 30$ & 0.0001 & 0.00045 & 0.0015 & 0.00035 & $(-0.0025,0.0034)$ \\
\hline $\cos (2 \pi t / 52)$ & 0.2 & 0.2103 & 0.0181 & 0.0103 & $(0.1748,0.2458)$ \\
\hline $\sin (2 \pi t / 52)$ & -0.4000 & -0.4253 & 0.0192 & 0.0253 & $(-0.4629,-0.3877)$ \\
\hline MA1 & -3 & -3.4125 & 0.0531 & 0.4125 & $(-3.5166,-3.3084)$ \\
\hline MA3 & -2 & -1.7526 & 0.0203 & 0.2474 & $(-1.7924,-1.7128)$ \\
\hline Intercept & 0.1 & 0.1811 & 0.0183 & 0.0811 & $(0.1452,0.2170)$ \\
\hline $\cos (2 \pi t / 52)$ & -0.4 & -0.4897 & 0.0216 & 0.0897 & $(-0.5320,-0.4474)$ \\
\hline $\sin (2 \pi t / 52)$ & -0.5 & -0.5612 & 0.0195 & 0.0612 & $(-0.5994,-0.5230)$ \\
\hline Overdisp & 2 & 2.8120 & 0.0636 & 0.8120 & $(2.6873,2.9367)$ \\
\hline \multicolumn{6}{|c|}{$\mathrm{N}=100$} \\
\hline Intercept & 0.3 & 0.2562 & 0.0180 & 0.0438 & $(0.2209,0.2914)$ \\
\hline$t / 100$ & 0.0001 & 0.00027 & 0.00039 & 0.00017 & $(-0.00049,0.0010)$ \\
\hline $\cos (2 \pi t / 52)$ & 0.2 & 0.2015 & 0.0096 & 0.0015 & $(0.1827,0.2202)$ \\
\hline $\sin (2 \pi t / 52)$ & -0.4000 & -0.4016 & 0.0097 & 0.0016 & $(-0.4207,-0.3826)$ \\
\hline MA1 & -3 & -3.1471 & 0.0220 & 0.1471 & $(-3.1902,-3.1040)$ \\
\hline MA3 & -2 & -2.1293 & 0.0169 & 0.1293 & $(-2.1624,-2.0961)$ \\
\hline Intercept & 0.1 & 0.1331 & 0.0100 & 0.0331 & $(0.1135,0.1527)$ \\
\hline $\cos (2 \pi t / 52)$ & -0.4 & -0.4748 & 0.0146 & 0.0748 & $(-0.5034,-0.4463)$ \\
\hline $\sin (2 \pi t / 52)$ & -0.5 & -0.5342 & 0.0136 & 0.0342 & $(-0.5609,-0.5075)$ \\
\hline Overdisp & 2 & 2.6211 & 0.0467 & 0.6211 & $(2.5296,2.7125)$ \\
\hline \multicolumn{6}{|c|}{$\mathrm{N}=500$} \\
\hline Intercept & 0.3 & 0.2851 & 0.0058 & 0.0149 & $(0.2737,0.2965)$ \\
\hline$t / 500$ & 0.0001 & 0.000101 & $1.8 \times 10^{-06}$ & $10^{-06}$ & $(0.00009,0.000104)$ \\
\hline $\cos (2 \pi t / 52)$ & 0.2 & 0.1966 & 0.0033 & 0.0034 & $(0.1902,0.2030)$ \\
\hline $\sin (2 \pi t / 52)$ & -0.4000 & -0.3983 & 0.0033 & 0.0017 & $(-0.4048,-0.3919)$ \\
\hline MA1 & -3 & -3.0117 & 0.0073 & 0.0117 & $(-3.0259,-2.9974)$ \\
\hline MA3 & -2 & -2.0098 & 0.0055 & 0.0098 & $(-2.0206,-1.9990)$ \\
\hline Intercept & 0.1 & 0.0918 & 0.0034 & 0.0082 & $(0.0851,0.0985)$ \\
\hline $\cos (2 \pi t / 52)$ & -0.4 & -0.4125 & 0.0048 & 0.0125 & $(-0.4220,-0.4030)$ \\
\hline $\sin (2 \pi t / 52)$ & -0.5 & -0.4993 & 0.0049 & 0.0007 & $(-0.5089,-0.4897)$ \\
\hline Overdisp & 2 & 2.1963 & 0.0124 & 0.1963 & $(2.1720,2.2205)$ \\
\hline \multicolumn{6}{|c|}{ MLE } \\
\hline \multicolumn{6}{|c|}{$\mathrm{N}=30$} \\
\hline Intercept & 0.3 & 0.3917 & 0.0288 & 0.0917 & $(0.3353,0.4481)$ \\
\hline$t / 30$ & 0.0001 & 0.00034 & 0.0027 & 0.00024 & $(-0.0050,0.0056)$ \\
\hline $\cos (2 \pi t / 52)$ & 0.2 & 0.2813 & 0.0191 & 0.0813 & $(0.2439,0.3187)$ \\
\hline $\sin (2 \pi t / 52)$ & -0.4 & -0.4513 & 0.0167 & 0.0513 & $(-0.4840,-0.4186)$ \\
\hline MA1 & -3 & -3.6412 & 0.0435 & 0.6412 & $(-3.7265,-3.5559)$ \\
\hline MA3 & -2 & -1.6513 & 0.0211 & 0.3487 & $(-1.6927,-1.6099)$ \\
\hline Intercept & 0.1 & 0.1884 & 0.0191 & 0.0884 & $(0.1510,0.2258)$ \\
\hline $\cos (2 \pi t / 52)$ & -0.4 & -0.4981 & 0.0254 & 0.0981 & $(-0.5479,-0.4483)$ \\
\hline $\sin (2 \pi t / 52)$ & -0.5 & -0.5815 & 0.0199 & 0.0815 & $(-0.6205,-0.5425)$ \\
\hline Overdisp & 2 & 2.9189 & 0.0731 & & $(2.7756,3.0621)$ \\
\hline \multicolumn{6}{|c|}{$\mathrm{N}=100$} \\
\hline Intercept & 0.3 & 0.3617 & 0.0197 & 0.0617 & $(0.3231,0.4003)$ \\
\hline$t / 100$ & 0.0001 & 0.0002 & 0.0003 & 0.0001 & $(-0.00038,0.00078)$ \\
\hline $\cos (2 \pi t / 52)$ & 0.2 & 0.2355 & 0.0100 & 0.0355 & $(0.2159,0.2551)$ \\
\hline $\sin (2 \pi t / 52)$ & -0.4 & -0.4060 & 0.0102 & 0.0060 & $(-0.4259,-3.860)$ \\
\hline MA1 & -3 & -3.1803 & 0.0240 & 0.1803 & $(-3.2273,-3.1332)$ \\
\hline MA3 & -2 & -2.1311 & 0.0184 & 0.1311 & $(-2.1673,-2.0950)$ \\
\hline Intercept & 0.1 & 0.1640 & 0.0185 & 0.0640 & $(0.1277,0.2002)$ \\
\hline $\cos (2 \pi t / 52)$ & -0.4 & -0.4538 & 0.0199 & 0.0538 & $(-0.4928,-0.4147)$ \\
\hline $\sin (2 \pi t / 52)$ & -0.5 & -0.5591 & 0.0222 & 0.0591 & $(-0.6026,-0.5155)$ \\
\hline Overdisp & 2 & 2.5507 & 0.0500 & 0.5507 & $(2.4527,2.6486)$ \\
\hline \multicolumn{6}{|c|}{$\mathrm{N}=500$} \\
\hline Intercept & 0.3 & 0.2862 & 0.0054 & 0.0138 & $(0.2756,0.2968)$ \\
\hline$t / 500$ & 0.0001 & 0.000102 & 0.00001 & $2 \times 10^{-06}$ & $(0.00008,0.00012)$ \\
\hline $\cos (2 \pi t / 52)$ & 0.2 & 0.2012 & 0.0029 & 0.0012 & $(0.1954,0.2069)$ \\
\hline $\sin (2 \pi t / 52)$ & -0.4 & -0.3954 & 0.0029 & 0.0046 & $(-0.4012,-0.3896)$ \\
\hline MA1 & -3 & -3.0192 & 0.0067 & 0.0192 & $(-3.0323,-3.0061)$ \\
\hline MA3 & -2 & -2.0140 & 0.0051 & 0.0140 & $(-2.0240,-2.0040)$ \\
\hline Intercept & 0.1 & 0.0922 & 0.0040 & 0.0078 & $(0.0844,0.0999)$ \\
\hline $\cos (2 \pi t / 52)$ & -0.4 & -0.4054 & 0.0053 & 0.0054 & $(-0.4158,-0.3950)$ \\
\hline $\sin (2 \pi t / 52)$ & -0.5 & -0.5013 & 0.0054 & 0.0013 & $(-0.5119,-0.4907)$ \\
\hline Overdisp & 2 & 2.1695 & 0.0118 & 0.1695 & $(2.1463,2.1927)$ \\
\hline
\end{tabular}




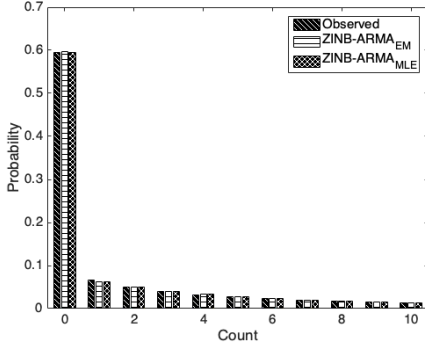

(a) Model 1

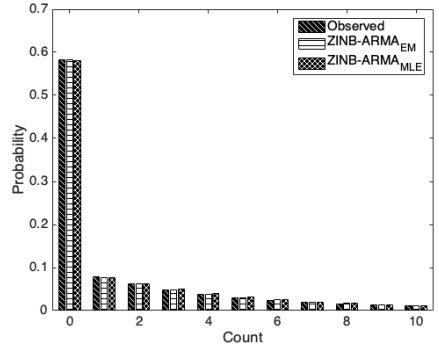

(b) Model 2

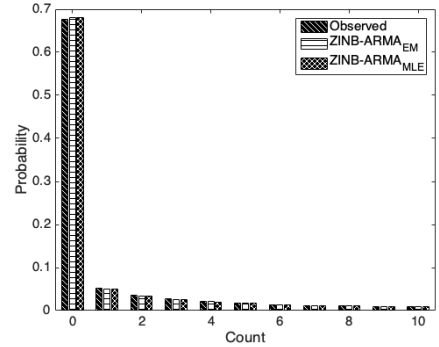

(c) Model 3

Figure 1: Goodness-of-fit plots for $n=100$ corresponding to simulation results of Models 1, 2 and 3 considered in Section 4. Here ZINB-ARMA denotes our proposed model of zero inflated NB-ARMA.
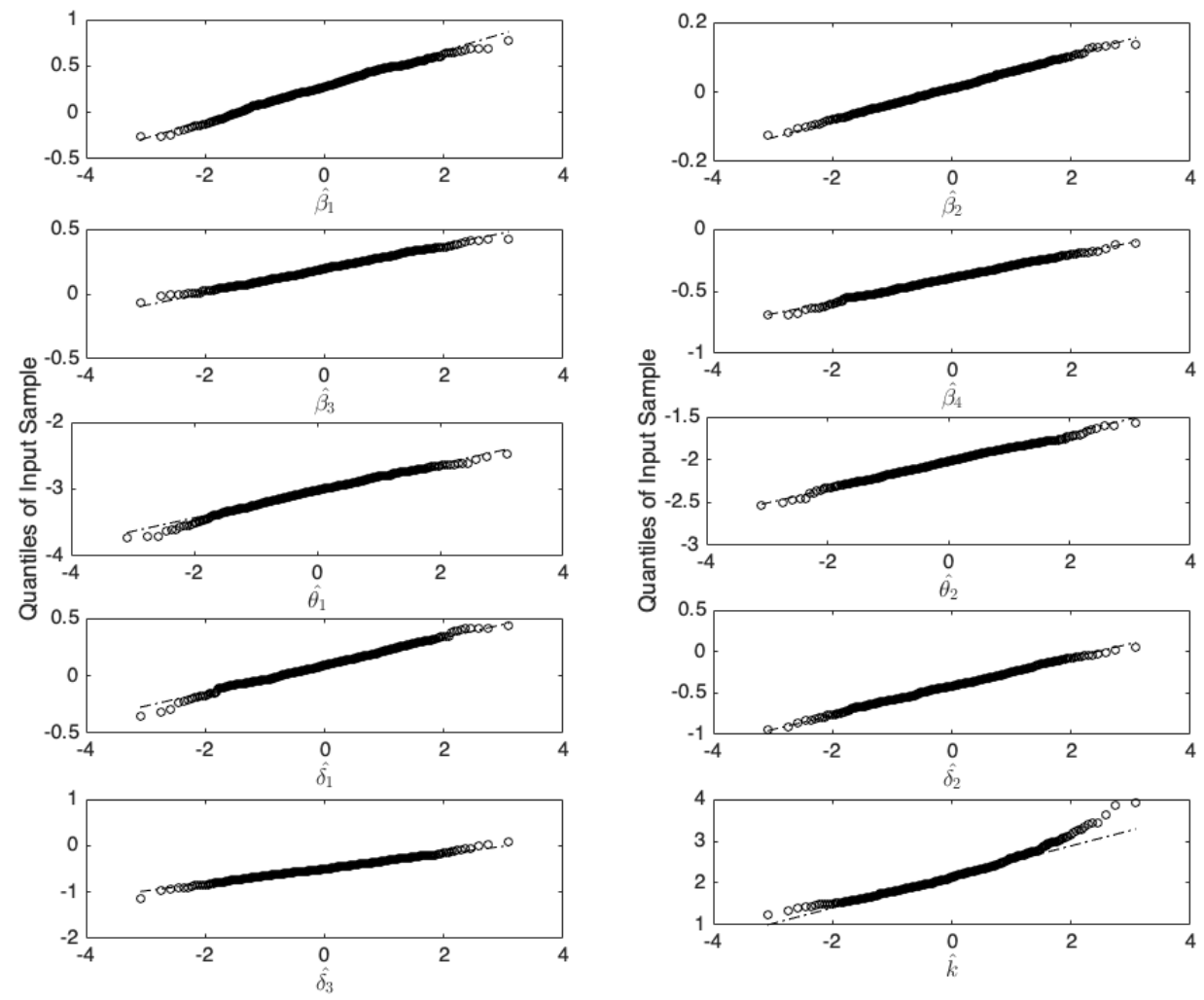

Figure 2: Normal probability plot i.e., QQ plot of the MLE estimates for simulated Model 3 in Section 4, $N=100$. Here $\beta_{1}=0.3, \beta_{2}=0.1, \beta_{3}=0.2, \beta_{4}=-0.4$, $\theta_{1}=-3, \theta_{2}=-2, \delta_{1}=0.1, \delta_{2}=-0.4, \delta_{3}=-0.5, k=2$. 
Table 2: Summary table for Models 1-6 in Section 5.2

\begin{tabular}{|c|c|c|c|c|c|c|c|}
\hline Model & \# parameters & MSE & $\chi^{2}$ & df & Deviance & AIC & BIC \\
\hline M1 & 16 & 9.4866 & 189.9052 & 133 & 108.2838 & 481.7745 & 529.8376 \\
M2 & 14 & 10.4535 & 199.0972 & 135 & 111.9753 & 485.1197 & 527.1749 \\
M3 & 16 & 10.5009 & 204.5800 & 133 & 112.0686 & 492.4039 & 540.4671 \\
M4 & 13 & 11.6191 & 200.7068 & 136 & 111.2048 & 485.0038 & 524.0551 \\
M5 & 10 & 12.9951 & 190.9382 & 139 & 109.8256 & 480.7847 & 510.8241 \\
M6 & 8 & 12.7397 & 189.2973 & 141 & 109.9701 & 485.9513 & 511.4829 \\
\hline
\end{tabular}

Table 3: EM Estimates of model M5 in Section 5.2

\begin{tabular}{|c|c|c|c|c|}
\hline Means & Parameters & Estimates & Std. Errors & p-values \\
\hline \multirow{6}{*}{$\lambda_{t}$} & Intercept & 2.0274 & 0.8010 & 0.0125 \\
& $t^{\prime} / 148$ & 1.2642 & 0.2866 & $<0.0001$ \\
& $\cos \left(2 \pi t^{\prime} / 52\right)$ & 0.9644 & 0.1812 & $<0.0001$ \\
& $\sin \left(2 \pi t^{\prime} / 52\right)$ & -1.8482 & 0.1508 & $<0.0001$ \\
& HMD & -0.0348 & 0.0108 & 0.0016 \\
& MA1 & -0.0935 & 0.0759 & 0.2197 \\
& MA3 & 0.1673 & 0.0575 & 0.0042 \\
& Intercept & -14.8254 & 8.6312 & 0.0881 \\
& cos $\left(2 \pi t^{\prime} / 52\right)$ & 12.9723 & 8.2209 & 0.1168 \\
& in $\left(2 \pi t^{\prime} / 52\right)$ & 6.8231 & 4.3688 & 0.1206 \\
\hline & overdisp. & 8.0569 & 3.1593 & 0.0118 \\
\hline
\end{tabular}

Table 4: Summary table for all compared models in Section 5.2

\begin{tabular}{|cccccc|}
\hline Model & \# Parameters & MSE & AIC & BIC & MAD \\
\hline M5 & 10 & 12.99 & 480.78 & 510.82 & 1.5315 \\
AM-1 & 9 & 13.64 & 487.76 & 517.74 & 1.7423 \\
AM-2 & 6 & 13.91 & 494.88 & 513.35 & 1.8156 \\
AM-3 & 4 & 16.19 & 863.14 & 899.19 & 2.4246 \\
AM-4 & 4 & 14.53 & 870.34 & 906.39 & 2.2270 \\
\hline
\end{tabular}

Table 5: Sensitivity and Specificity Values for M5 and AM-1 considered in Section 5.2. "Th" represents threshold.

\begin{tabular}{|c|c|c|c|c|c|c|}
\hline Model & \multicolumn{2}{|c|}{ Th=0.4 } & \multicolumn{2}{c|}{ Th=0.5 } & \multicolumn{2}{c|}{ Th=0.6 } \\
\hline & Sensitivity & Specificity & Sensitivity & Specificity & Sensitivity & Specificity \\
\hline M5 & $92 \%$ & $85 \%$ & $100 \%$ & $83 \%$ & $100 \%$ & $81 \%$ \\
AM-1 & $60 \%$ & $85 \%$ & $68 \%$ & $81 \%$ & $74 \%$ & $78 \%$ \\
\hline
\end{tabular}



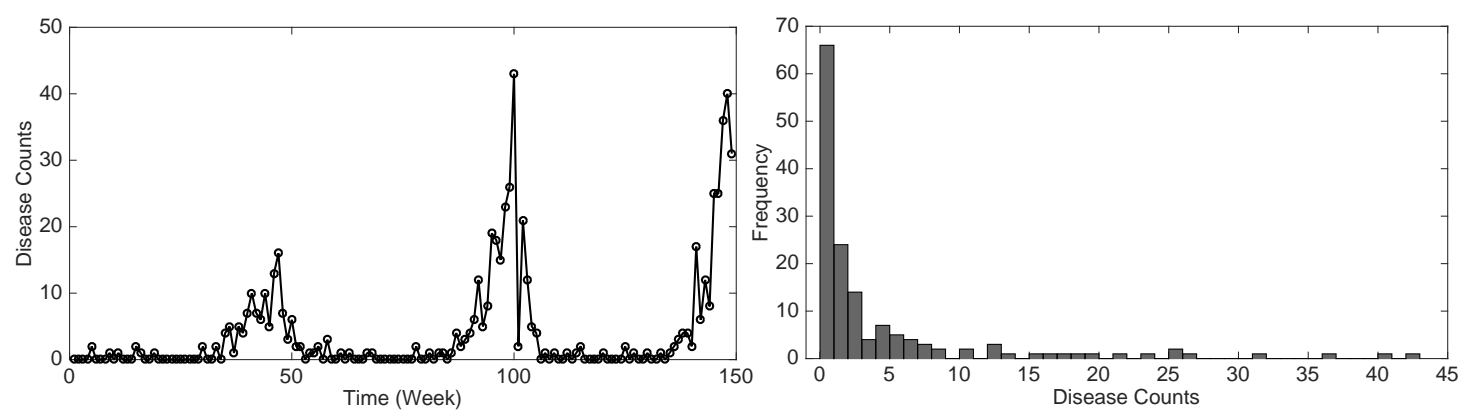

Figure 3: Time Series plot and histogram of weekly dengue count data

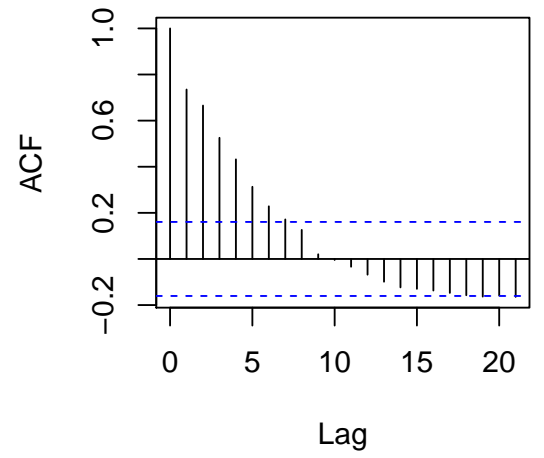

(a) ACF plot

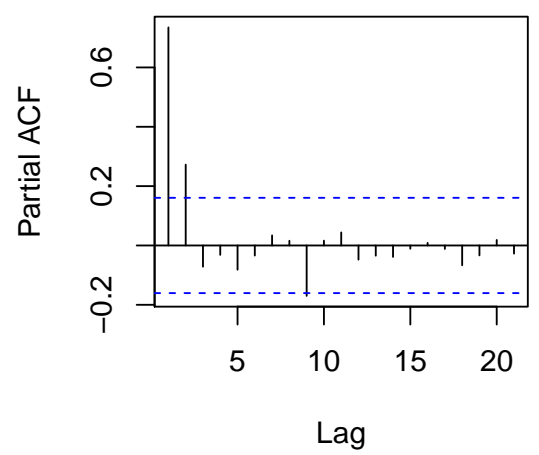

(b) PACF plot

Figure 4: ACF and PACF plots of raw weekly dengue counts

Table 6: Summary table for Models 1-6 corresponding to dengue data in Section 6

\begin{tabular}{|c|c|c|c|c|c|c|c|}
\hline Model & \# parameters & MSE & $\chi^{2}$ & df & Deviance & AIC & BIC \\
\hline M1 & 4 & 20.7445 & 190.8015 & 205 & 143.3237 & 1080.6572 & 1097.3698 \\
M2 & 5 & 20.1038 & 190.5589 & 204 & 144.3150 & 1075.0041 & 1091.7157 \\
M3 & 6 & 19.8279 & 188.6323 & 203 & 144.7702 & 1072.6851 & 1092.5425 \\
M4 & 7 & 19.9974 & 188.6969 & 202 & 144.5563 & 1079.1456 & 1092.7394 \\
M5 & 6 & 20.2616 & 191.2593 & 203 & 144.6008 & 1077.9771 & 1098.0313 \\
M6 & 7 & 19.8713 & 188.8358 & 202 & 144.9468 & 1075.3745 & 1098.7672 \\
\hline
\end{tabular}




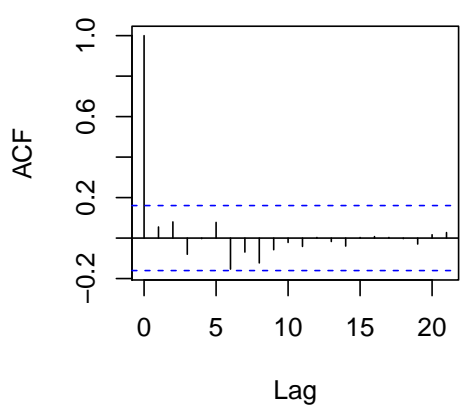

(a) ACF plot of quantile residuals

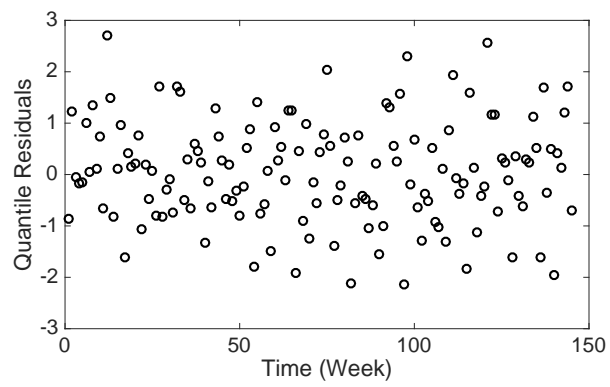

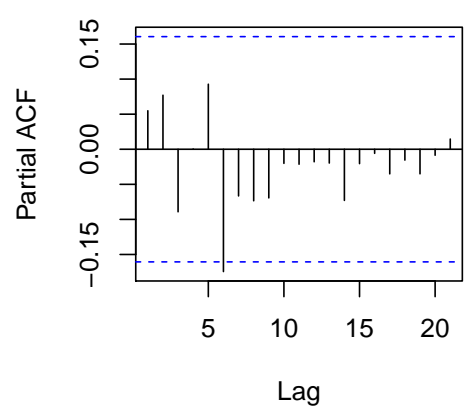

(b) PACF plot of quantile residuals

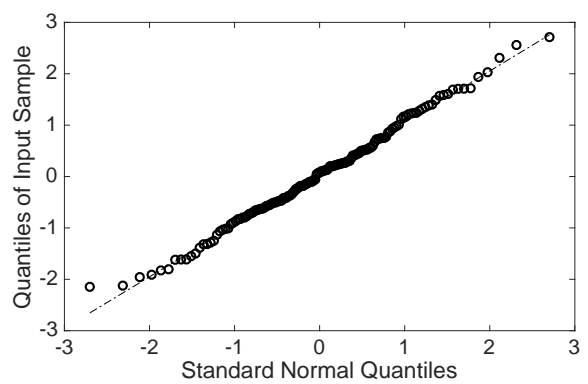

(c) Randomized quantile residuals and QQ-plot

Figure 5: Randomized quantile residual analysis for model M5 in Section 5.2

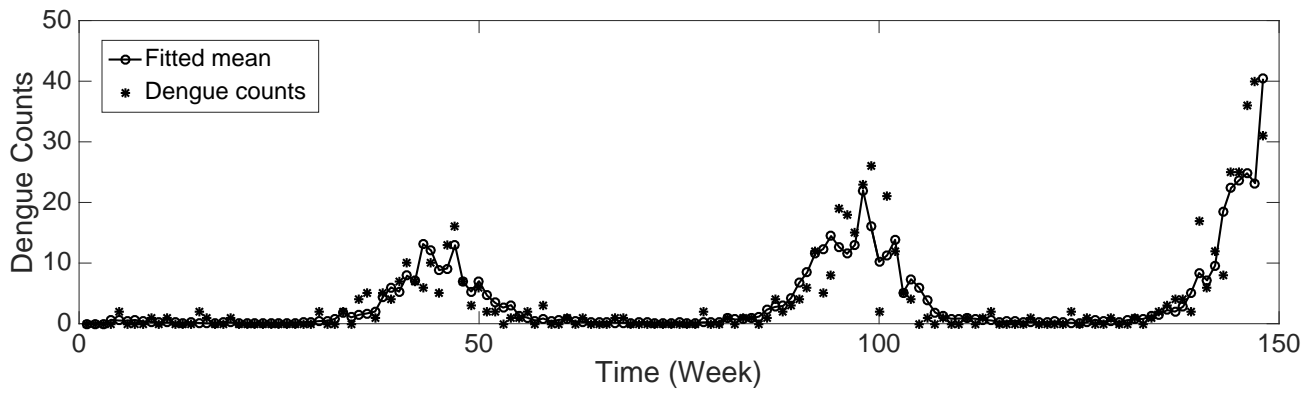

Figure 6: Actual dengue counts and fitted mean values from Model 5 
Table 7: EM estimates of zero-inflated NB-ARMA fitted to Syphilis data, in Section 6

\begin{tabular}{|c|c|c|c|c|}
\hline Means & Parameters & Estimates & Std. Errors & p-values \\
\hline \multirow{3}{*}{$\lambda_{t}$} & Intercept & 1.6134 & 0.0949 & 0 \\
& $t^{\prime} / 208$ & 0.3775 & 0.1564 & 0.0167 \\
& MA1 & -0.1509 & 0.0608 & 0.0138 \\
& MA2 & -0.0724 & 0.0562 & 0.1995 \\
\hline \multirow{2}{*}{$\pi_{t}$} & Intercept & -1.3091 & 0.3724 & 0.0005 \\
& $t^{\prime} / 208$ & 0.2122 & 0.6121 & 0.7291 \\
\hline & overdisp. & 3.0981 & 0.6864 & 0.0000 \\
\hline
\end{tabular}

Table 8: Model comparisons for Syphilis data, in Section 6

\begin{tabular}{|c|c|c|c|}
\hline Model & MSE & AIC & BIC \\
\hline Zero inflated NB-ARMA & 19.8279 & 1072.6851 & 1092.5425 \\
ZIP & 20.2455 & 1173.6862 & 1187.0551 \\
ZINB & 20.2326 & 1077.3358 & 1097.3898 \\
FRM Method & 20.2468 & 1078.3750 & 1098.4290 \\
\hline
\end{tabular}

